\title{
Geometric estimates for the trace formula
}

\author{
Werner Hoffmann
}

September 28, 2005

\begin{abstract}
In order to study the asymptotic distribution of geometric or spectral data associated with quotients of a reductive group by a lattice, one needs a trace formula for test functions on that group with noncompact support. Arthur has proved a trace formula for compactly supported test functions on reductive groups of arbitrary rank. We show that the coarse geometric expansion in his formula converges for rapidly decreasing functions.
\end{abstract}

Mathematics Subject Classification. Primary: 11F72; Secondary: 22E55.

Keywords and phrases. Trace formula, Harish-Chandra's Schwartz space, truncation.

\section{Introduction}

Let $G$ be a connected reductive group defined over a global field $F$ and denote, for each $F$-algebra $E$, by $G(E)$ the group of $E$-valued points. The diagonal embedding of $F$ into its adele ring $\mathbb{A}$ gives rise to an embedding of $G(F)$ into the locally compact group $G(\mathbb{A})$ as a discrete subgroup. Thus we can consider the space of square-integrable functions on $G(F) \backslash G(\mathbb{A})$ with respect to an invariant measure. The unitary representation $R$ of $G(\mathbb{A})$ acting on this space by right translations can be integrated with the aid of a Haar measure to give a representation of the algebra $L^{1}(G(\mathbb{A}))$. The trace formula is one of the main tools to study the decomposition of the discrete part of this representation, the notion of discrete having been suitably modified if $G(\mathbb{A})$ has noncompact centre. In case $G$ is anisotropic and $f$ is a smooth compactly supported function on $G(\mathbb{A})$, this formula expresses the trace of $R(f)$ in terms of geometric data. For general $G$, one still has an identity between two distributions, called the spectral and the geometric expansion, respectively [1], [2]. The trace of the discrete part of $R(f)$ is among the spectral terms, at least in the cases where a certain convergence problem has been resolved [20].

In some applications of the trace formula, specifically if one asks for the asymptotic distribution of geometric or spectral data associated with the space 
$G(F) \backslash G(\mathbb{A})$, it is awkward or even impossible to get by with test functions $f$ of compact support. One might expect that the trace formula is valid for $f$ in Harish-Chandra's Schwartz space $\mathcal{C}^{1}\left(G\left(F_{S}\right)\right)$ for a finite set $S$ of places of $F$, where $F_{S}:=\prod_{v \in S} F_{v}$ and $f$ is suitably extended to $G(\mathbb{A})$. The present paper is devoted to the proof that the coarse geometric expansion of the trace formula, as described in [1], is in fact absolutely convergent for test functions in the Schwartz space $\mathcal{C}^{p}\left(G\left(F_{S}\right)\right)$ for $p$ small enough. Actually, we show not only the convergence of an integral, but the rapid decay at infinity of its integrand. By inspection of our proof one may derive an explicit, although not optimal, lower bound on the best possible $p$ for a given group $G$.

The operator $R(f)$ is an integral operator with kernel $K(x, y)$. If $G$ is anisotropic, then the trace can be obtained by integrating this kernel over the diagonal. In general, one has to form an alternating sum of kernels $K^{P}(x, y)$ multiplied with characteristic functions of certain sets. These sets depend on a so-called truncation parameter $T$, which runs through an affine space. In [1], Arthur has proved the convergence for sufficiently regular $T$ depending on the support of $f$. In our situation, this support could be all of $G(\mathbb{A})$, and then no parameter $T$ would be sufficiently regular. In order to solve this problem, we give a different argument, which works for all $T$. The resulting geometric side of the trace formula depends polynomially on $T$ (with a slight modification in case of positive characteristic), as already proved in [3] in the sufficiently regular range. The previous lack of knowledge that the explicit expression is convergent for all $T$ was the source of certain complications in [4], which may now be avoided, at least for compactly supported $f$. However, further insight will be required to extend the fine geometric expansion of the trace formula to test functions of noncompact support.

\section{The results}

Let $F, \mathbb{A}$ and $G$ be as in the introduction. If $G(\mathbb{A})$ has noncompact centre, then $R(f)$ is never of trace class. This is why one often considers functions on $G(F) \backslash G(\mathbb{A})$ transforming under the centre according to a fixed central character. We follow the alternative approach of $[1]$ and replace $G(\mathbb{A})$ by the subgroup $G(\mathbb{A})^{1}$ consisting of all $g$ such that $|\chi(g)|=1$ for every rational character $\chi$ of $G$ defined over $F$. Note that this notation makes sense if we replace $G$ by any linear algebraic group defined over a field $F$ and $\mathbb{A}$ by any locally-compact $F$-algebra, where $|a|$ denotes the module of $a \in \mathbb{A}^{\times}$with respect to any Haar measure on $\mathbb{A}^{\times}$.

In order to state the definition of the spectral expansion of the trace formula, we first have to recall some notation. For each parabolic $F$-subgroup $P$ of $G$ with unipotent radical $N$, we have a representation $R^{P}$ of $G(\mathbb{A})^{1}$ on $L^{2}\left(P(F) N(\mathbb{A}) \backslash G(\mathbb{A})^{1}\right)$ by right translations. Fixing $f \in L^{1}\left(G(\mathbb{A})^{1}\right)$ and integrating $f(g) R^{P}(g)$ with respect to a Haar measure of $G(\mathbb{A})^{1}$, we obtain an integral 
operator with kernel given by

$$
K^{P}(x, y)=\int_{N(F) \backslash N(\mathbb{A})} \sum_{\gamma \in P(F)} f\left(x^{-1} \gamma n y\right) d n
$$

for almost every $x, y \in G(F) \backslash G(\mathbb{A})^{1}$, which could also be written as an integral over $P(F) N(\mathbb{A})$ and whose convergence is deduced from Fubini's theorem. All invariant measures on coset spaces are chosen such that they satisfy the obvious compatibility conditions. As usual, one omits the superscript $P$ if $P=G$.

The coarse geometric expansion of these kernels comes from an equivalence relation on $G(F)$ defined as the finest equivalence relation with the following properties:

(a) Any two $G(F)$-conjugate elements are equivalent.

(b) If $P$ is a parabolic $F$-subgroup with unipotent radical $N$, then the elements of each $N(F)$-coset in $P(F)$ are mutually equivalent.

Let $\mathcal{O}$ be the set of equivalence classes. If $M$ is a Levi component of $P$ defined over $F$, we can restate condition (b) as

$$
P(F) \cap \mathfrak{o}=(M(F) \cap \mathfrak{o}) N(F)
$$

for any $\mathfrak{o} \in \mathcal{O}$. It is sufficient to require this for minimal $P$ such that $P(F) \cap \mathfrak{o} \neq \emptyset$. In this case, any $\gamma \in M(F) \cap \mathfrak{o}$ is $F$-elliptic in $M$ (i.e., not contained in any proper parabolic $F$-subgroup of $M)$. One can check that in this way one obtains a bijection between $\mathcal{O}$ and the set of $G(F)$-conjugacy classes of pairs $(M, \gamma)$, where $M$ is a Levi $F$-subgroup of $G$ and $\gamma$ is an $F$-elliptic element of $M(F)$.

In the case char $F=0$, there is an alternative description of our equivalence relation, which was taken for the definition in [1]. Namely, one can show that two elements of $G(F)$ are equivalent iff the semisimple components in their Jordan decompositions are $G(F)$-conjugate. The remark after the proof of Lemma 2.1 in [1] proves this claim in one direction.

Deferring questions of convergence, we write $K^{P}(x, y)$ as the sum over all $\mathfrak{o} \in \mathcal{O}$ of the partial kernels

$$
K_{\mathfrak{o}}^{P}(x, y)=\int_{N(F) \backslash N(\mathbb{A})} \sum_{\gamma \in P(F) \cap \mathfrak{o}} f\left(x^{-1} \gamma n y\right) d n,
$$

which are well defined due to condition (b). Such a partial kernel is not $P(F)$ invariant as a function of $x$ or $y$ in general, but the function of one variable obtained by setting $x=y$ is.

Recall that $\mathbb{A}$ is the restricted direct product of all completions $F_{v}$ with respect to the places $v$ of $F$. Taking the partial product over the archimedean and nonarchimedean places, resp., we obtain direct product decompositions $\mathbb{A}=F_{\infty} \times$ 
$\mathbb{A}_{\mathrm{f}}$ and $G(\mathbb{A})=G\left(F_{\infty}\right) G\left(\mathbb{A}_{\mathrm{f}}\right)$. The truncation procedure requires fixing a maximal compact subgroup $K$ of $G(\mathbb{A})$. Such groups are of the form $K=\prod_{v} K_{v}$, where $K_{v}$ is a maximal compact subgroup in $G\left(F_{v}\right)$. We assume that the partial product $K_{\mathrm{f}}$ over all nonarchimedian places is an open subgroup of $G\left(\mathbb{A}_{\mathrm{f}}\right)$. Then $G(\mathbb{A})$ is the restricted direct product of the groups $G\left(F_{v}\right)$ with respect to the subgroups $K_{v}$, and $K_{v}$ is a special maximal compact subgroup for almost all $v$. We further assume that the latter is true for all nonarchimedean $v$. Then one has $G(\mathbb{A})=$ $P(\mathbb{A}) K$ for any parabolic $F$-subgroup $P$ of $G$.

For each such $P$, let $X(P)$ be the group of all $F$-rational characters of $P$ and $\delta_{P} \in X(P)$ the determinant of the adjoint action of $P$ on the Lie algebra of $N$. We define the real vector space $\mathfrak{a}_{P}=\operatorname{Hom}(X(P), \mathbb{R})$ and the map $H_{P}: G(\mathbb{A}) \rightarrow \mathfrak{a}_{P}$ by $H_{P}(p k)=H_{P}(p): \chi \mapsto \log |\chi(p)|$ for every $p \in P(\mathbb{A}), k \in K$ and $\chi \in X(P)$. These maps are compatible with the natural projections $\mathfrak{a}_{P} \rightarrow \mathfrak{a}_{P^{\prime}}$ for $P \subset P^{\prime}$. The cone ${ }^{+} \mathfrak{a}_{P}$ can be defined as the set of all $H \in \mathfrak{a}_{P}$ which take positive values on $\delta_{P^{\prime}}$ for all proper parabolic subgroups $P^{\prime}$ of $G$ containing $P$. The kernel of $H_{P}$ is $P(\mathbb{A})^{1}$.

Now we are ready to define the geometric expansion of the trace formula. It is customary (although not necessary, see [6], p. 271) to fix a minimal parabolic $F$-subgroup $P_{0}$. For $\mathfrak{o} \in \mathcal{O}, T \in \mathfrak{a}_{0}:=\mathfrak{a}_{P_{0}}$ and $x \in G(\mathbb{A})^{1}$, we set

$$
k_{\mathfrak{o}}^{T}(x)=\sum_{P} \varepsilon_{P}^{G} \sum_{\delta \in P(F) \backslash G(F)} K_{\mathfrak{o}}^{P}(\delta x, \delta x) \hat{\tau}_{P}\left(H_{P}(\delta x)-T_{P}\right),
$$

the exterior sum being taken over all parabolic subgroups containing $P_{0}$. Here $\varepsilon_{P}^{G}=(-1)^{\operatorname{dim} \mathfrak{a}_{P} / \mathfrak{a}_{G}}, T_{P}$ is the projection of $T$ on $\mathfrak{a}_{P}$, and $\hat{\tau}_{P}$ is the characteristic function of ${ }^{+} \mathfrak{a}_{P}$. The coarse geometric expansion of the trace formula, introduced in [1], is

$$
J^{T}(f)=\sum_{\mathfrak{o} \in \mathcal{O}} \int_{G(F) \backslash G(\mathbb{A})^{1}} k_{\mathfrak{o}}^{T}(x) d x .
$$

(It is parallel to the coarse spectral expansion in terms of $G(F)$-conjugacy classes of pairs $(M, \pi)$, where $M$ is a Levi $F$-subgroup of $G$ and $\pi$ is a cuspidal automorphic representation of $M(\mathbb{A})$.) Of course, it has to be shown that all sums and integrals occurring here are absolutely convergent.

We are going to prove this when $f$ is a suitable extension of a function in a certain Schwartz space $\mathcal{C}^{p}\left(G\left(F_{S}\right)^{1}\right)$, which has been introduced in the case $p=2$ in [3], p. 28, and is some kind of tensor product of the spaces introduced by Harish-Chandra. Namely, let $\Xi_{v}$ and $\sigma_{v}$ be the functions on $G\left(F_{v}\right)$ used in [11] and [13] in the definition of $\mathcal{C}\left(G\left(F_{v}\right)\right.$ ) (cf. section 3 for details). Given a finite set $S$ of places of $F$, we set, for any $g \in G\left(F_{S}\right)$,

$$
\Xi_{S}(g)=\prod_{v \in S} \Xi_{v}\left(g_{v}\right), \quad \sigma_{S}(g)=\prod_{v \in S} \sigma_{v}\left(g_{v}\right) .
$$


Let $S=S_{\infty} \cup S_{\mathrm{f}}$ be the decomposition into archimedean and non-archimedean places. A function on $G\left(F_{S}\right)^{1}$ is called smooth if it is biinvariant under some open compact subgroup $K_{0}$ of $G\left(F_{S_{\mathrm{f}}}\right)$ and is infinitely differentiable as a function on the manifold $K_{0} \backslash G\left(F_{S}\right)^{1} / K_{0}$. The actions of the Lie group $G\left(F_{S_{\infty}}\right)^{1}$ on $G\left(F_{S}\right)^{1}$ by left and right translations give rise to representations $X \mapsto X_{L}, X \mapsto X_{R}$ of its Lie algebra on the space of smooth functions, which extend to the universal enveloping algebra $\mathfrak{G}_{S}^{1}$. (If $S_{\infty}=\emptyset$, then $G\left(F_{S_{\infty}}\right)^{1}=\{1\}$ and $\mathfrak{G}_{S}^{1}=\mathbb{C}$ ). For all $p>0, r>0$ and finite sets $\mathfrak{F} \subset \mathfrak{G}_{S}^{1} \times \mathfrak{G}_{S}^{1}$ we set

$$
\|f\|_{p, \mathfrak{F}, r}=\max _{(X, Y) \in \mathfrak{F}} \sup _{g \in G\left(F_{S}\right)^{1}}\left|X_{L} Y_{R} f(g)\right| \Xi_{S}(g)^{-2 / p}\left(1+\sigma_{S}(g)\right)^{r} .
$$

We define $\mathcal{C}_{K_{0}}^{p}\left(G\left(F_{S}\right)^{1}\right)$ as the space of all $K_{0}$-biinvariant smooth functions $f$ on $G\left(F_{S}\right)^{1}$ such that $\|f\|_{p, \mathfrak{F}, r}<\infty$ for all $\mathfrak{F}$ and $r$. The seminorms $\|\cdot\|_{p, \mathfrak{F}, r}$ induce a topology on $\mathcal{C}_{K_{0}}^{p}\left(G\left(F_{S}\right)^{1}\right)$, and we define $\mathcal{C}^{p}\left(G\left(F_{S}\right)^{1}\right)$ as the topological direct limit, over all $K_{0}$, of the spaces $\mathcal{C}_{K_{0}}^{p}\left(G\left(F_{S}\right)^{1}\right)$. It is known (see section 3) that this space and its topology do not depend on the choice of $K_{S}=\prod_{v \in S} K_{v}$ and that $\mathcal{C}^{p}\left(G\left(F_{S}\right)^{1}\right) \subset L^{p}\left(G\left(F_{S}\right)^{1}\right)$, the embedding being continuous.

Any function in $\mathcal{C}^{p}\left(G\left(F_{S}\right)^{1}\right)$ can be uniquely extended to a $K^{S}$-invariant function on $G\left(F_{S}\right)^{1} K^{S}$, where $K^{S}=\prod_{v \notin S} K_{v}$. The resulting space will be denoted by $\mathcal{C}^{p}\left(G\left(F_{S}\right)^{1} K^{S}\right)$, and such functions will tacitly be extended to $G(\mathbb{A})^{1}$ by setting them equal to zero outside $G\left(F_{S}\right)^{1} K^{S}$. The notations $\|f\|_{p, \mathfrak{F}, r}$ for such functions and $\mathcal{C}_{K_{0}}^{p}\left(G\left(F_{S}\right)^{1} K^{S}\right)$ have the obvious meaning. We will always assume that $S$ contains all archimedean places (if any), so these functions are smooth on $G(\mathbb{A})^{1}$, and $\mathfrak{G}^{1}=\mathfrak{G}_{S}^{1}$ is independent of $S$.

Remark. Both functions $\Xi_{v}$ and $\sigma_{v}$ take value 1 on $K_{v}$. Thus, for $S \subset S^{\prime}$, the restrictions of the seminorms of $\mathcal{C}^{p}\left(G\left(F_{S^{\prime}}\right)^{1} K^{S^{\prime}}\right)$ to $\mathcal{C}^{p}\left(G\left(F_{S}\right)^{1} K^{S}\right)$ coincide with the corresponding seminorms of the latter space. Consequently, the topological direct limit $\mathcal{C}^{p}\left(G(\mathbb{A})^{1}\right)$ of these spaces over all $S$ is just their union, and it is independent of $K$, because for any two maximal compact subgroups $K$ and $K^{\prime}$ satisfying our assumptions we have $K_{v}=K_{v}^{\prime}$ for almost all $v$.

Theorem 1 There exists $p>0$ with the following property. For every $S$ containing all infinite places, every open compact subgroup $K_{0}$ of $G\left(F_{S_{\mathrm{f}}}\right)$ and every truncation parameter $T$ there exist a constant $r$ and a finite set $\mathfrak{F} \subset \mathfrak{G}^{1} \times \mathfrak{G}^{1}$ such that

$$
\sum_{\mathfrak{o} \in \mathcal{O}} \int_{G(F) \backslash G(\mathbb{A})^{1}}\left|k_{\mathfrak{o}}^{T}(x)\right| d x \leq\|f\|_{p, \mathfrak{F}, r}
$$

for all $f \in \mathcal{C}_{K_{0}}^{p}\left(G\left(F_{S}\right)^{1} K^{S}\right)$.

Note that $k_{\mathfrak{o}}^{T}$ depends implicitly on $f$. The ostensibly missing constant factor has been absorbed into $\mathfrak{F}$. 
This theorem is our main result, which generalises Theorem 7.1 of [1] by removing the assumptions that $f$ have compact support, $T$ be sufficiently regular and $F$ be a number field.

As already mentioned, Theorem 1 may be strengthened to a bound on $k_{\mathfrak{o}}^{T}$. This function being left $G(F)$-invariant, it is sufficient to bound its restriction to a Siegel domain $\mathcal{S}_{0}^{1}$ with respect to $P_{0}$ satisfying $G(F) \mathcal{S}_{0}^{1}=G(\mathbb{A})^{1}$, cf. [10]. (The definition of a Siegel domain will be recalled in section 6.) Let us write $\rho_{0}=\rho_{P_{0}}$ and $H_{0}=H_{P_{0}}$.

Theorem 2 There exist $c>0$ and $d$ with the following property. For every $S$ containing all infinite places, every open compact subgroup $K_{0}$ of $G\left(F_{S_{\mathrm{f}}}\right)$, every truncation parameter $T$ and $0<p<1$ there exist a constant $r$ and a finite set $\mathfrak{F} \subset \mathfrak{G}^{1} \times \mathfrak{G}^{1}$ such that

$$
\sum_{\mathfrak{o} \in \mathcal{O}}\left|k_{\mathfrak{o}}^{T}(x)\right| \leq\|f\|_{p, \mathfrak{F}, r} e^{(d-c / p) \rho_{0}\left(H_{0}(x)\right)}
$$

for all $f \in \mathcal{C}_{K_{0}}^{p}\left(G\left(F_{S}\right)^{1} K^{S}\right)$ and $x \in \mathcal{S}_{0}^{1}$.

The method to prove this theorem goes back to an old preprint named "The decay of truncated kernels on locally-symmetric spaces", where I estimated the non-adelic analog of the truncated kernel

$$
k^{T}(x, y)=\sum_{P} \varepsilon_{P}^{G} \sum_{\delta, \eta \in P(F) \backslash G(F)} K^{P}(\delta x, \eta y) \hat{\tau}_{P}\left(H_{P}(\delta x)-T_{P}\right) \hat{\tau}_{P}\left(H_{P}(\eta y)-T_{P}\right)
$$

without restricting it to the diagonal. Since $k^{T}(x, x)$ does not coincide with $\sum_{\mathfrak{o}} k_{\mathfrak{o}}^{T}(x)$ unless all factors of $G$ have $F$-rank one, and since it is impossible, for $x \neq y$, to separate the contribution from various classes $\mathfrak{o}$, the old result does not imply Theorem 1. It was intended as the first step in extending Faddeev's method of analytic continuation of (cuspidal) Eisenstein series ([7], cf. also ch. XIV of [15]) to the higher rank case. However, it never appeared in a journal as I was unable to generalise the boudary conditions required for Faddeev's method, while Jacquet's elegant simplification of Selberg's original proof, as exposed in ch. IV of [19], made the search for alternative methods pointless. Nevertheless, I include that kernel estimate here as it might still have other applications and its proof is a byproduct of the arguments we have to expound anyway.

Theorem 3 There exist $c>0$ and $d$ with the following property. For every $S$ containing all infinite places, every open compact subgroup $K_{0}$ of $G\left(F_{S_{\mathrm{f}}}\right)$, every truncation parameter $T, 0<p<1$ and large $r$ there exists a finite set $\mathfrak{F} \subset \mathfrak{G}^{1} \times \mathfrak{G}^{1}$ such that

$$
\left|k^{T}(x, y)\right| \leq\|f\|_{p, \mathfrak{F}, r} e^{(d-c / p) \rho_{0}\left(H_{0}(x)+H_{0}(y)\right)}
$$

for $f \in \mathcal{C}_{K_{0}}^{p}\left(G\left(F_{S}\right)^{1} K^{S}\right)$ and $x, y \in \mathcal{S}_{0}^{1}$. 


\section{Preliminaries concerning root systems}

In this section we collect some easy facts needed later. We consider a connected reductive $F$-group $G$ like in section 1 , but now $F$ can be any field.

Let $A$ be an $F$-split torus in $G$ and $M$ its centraliser. We call $A$ a special $F$-torus in $G$ if it is the largest $F$-split torus in the centre of $M$. If this is the case, $M$ is a Levi component of some parabolic $F$-subgroup of $G$ or, as we shall say for brevity, a Levi $F$-subgroup of $G$. In this way one gets a bijection between the sets of special $F$-tori and of Levi $F$-subgroups of $G$.

Let $X(A)$ denote the group of $F$-rational characters of the special $F$-torus $A$, set $\mathfrak{a}=\operatorname{Hom}(X(A), \mathbb{R})$ (the "real Lie algebra" of $A$ ) and denote by $\Sigma_{A} \subset X(A)$ the set of roots of $A$ in $G$. Following tradition, we often write the group $X(A)$ additively and consider its elements as linear functionals on $\mathfrak{a}$. A subspace of $\mathfrak{a}$ is called a special subspace if it is the intersection of the kernels of some elements from $\Sigma_{A}$. If $A^{\prime}$ is a special $F$-torus contained in $A$, then we have a natural map $\mathfrak{a}^{\prime} \rightarrow \mathfrak{a}$. All such maps are compatible, which allows us to identify $\mathfrak{a}^{\prime}$ with a subspace of $\mathfrak{a}$. In this way we get a bijection from the set of special $F$-tori contained in $A$ onto the set of special subspaces of $\mathfrak{a}$. Let $\mathfrak{a}_{\text {reg }}$ denote the set of all $H \in \mathfrak{a}$ which are not contained in any special subspace except $\mathfrak{a}$ itself. The connected components of $\mathfrak{a}_{\text {reg }}$ are called the chambers of $\mathfrak{a}$.

For $A$ and $M$ as above, let $\mathcal{P}(A)$ be the set of parabolic $F$-subgroups of $G$ having $M$ as a Levi component. For $P \in \mathcal{P}(A)$, the space $\mathfrak{a}_{P}$ introduced in section 1 depends on $P$ but not on $A$, and is naturally isomorphic to $\mathfrak{a}$. The set of roots of $A$ in the unipotent radical of $P$, which we denote by $\Sigma_{P}$, may be identified with a subset of $\mathfrak{a}_{P}^{*}$ (which justifies the absence of $A$ in the notation), and we can regard $\rho_{P}:=\frac{1}{2} \delta_{P}$ as a linear functional on $\mathfrak{a}$. Although $\mathfrak{a}$ figured as a universal variable in the symbol $\mathfrak{a}_{P}$, in the presence of a special split torus the corresponding lower-case gothic letter will henceforth refer to its real Lie algebra. E.g., we denote by $\mathfrak{a}_{P}^{+}$the set of all $H \in \mathfrak{a}$ which take positive values on the elements of $\Sigma_{P}$, and we also regard ${ }^{+} \mathfrak{a}_{P}$ as a subset of $\mathfrak{a}$. Thus, by assigning $\mathfrak{a}_{P}^{+}$ to $P$, we get a bijection of $\mathcal{P}(A)$ onto the set of chambers in $\mathfrak{a}$.

Lemma 1 If $A$ is a special $F$-torus and $H \in \mathfrak{a}$, set

$$
|H|=\sup _{P \in \mathcal{P}(A)} \rho_{P}(H)
$$

(i) This is a seminorm on $\mathfrak{a}$ vanishing exactly on $\mathfrak{a}_{G}$, and for every $P \in \mathcal{P}(A)$ we have

$$
|H|=\frac{1}{4} \sum_{\alpha \in \Sigma_{A}} m_{\alpha}|\alpha(H)|=\frac{1}{2} \sum_{\alpha \in \Sigma_{P}} m_{\alpha}|\alpha(H)|,
$$

where $m_{\alpha}$ is the multiplicity of $\alpha$. Moreover, $|H|=\rho_{P}(H)$ iff $H \in \overline{\mathfrak{a}}_{P}^{+}$. 
(ii) Suppose $G$ and $A$ are also defined over a subfield $F^{\prime}$ of $F$ and that $A^{\prime}$ is a special $F^{\prime}$-torus in $G$ contained in $A$. Let $M^{\prime}$ be the centraliser of $A^{\prime}$ and define $X^{\prime}\left(A^{\prime}\right), \mathfrak{a}^{\prime}$ and $|.|^{\prime}$ with respect to the ground field $F^{\prime}$. Then the inclusion $\mathfrak{a}^{\prime} \rightarrow \mathfrak{a}$ dual to the restriction map $X(A) \rightarrow X\left(A^{\prime}\right) \cong X^{\prime}\left(A^{\prime}\right)$ is an isometric embedding, and the projection $\mathfrak{a} \rightarrow \mathfrak{a}^{\prime}, H \mapsto H^{\prime}$, dual to the restriction map $X^{\prime}\left(M^{\prime}\right) \rightarrow X(M)$ satisfies $\left|H^{\prime}\right|^{\prime} \leq|H|$.

Proof. Fix $H \in \mathfrak{a}$ and choose $Q \in \mathcal{P}(A)$ such that $H \in \overline{\mathfrak{a}_{Q}^{+}}$. Then

$$
\rho_{P}(H)=\frac{1}{2} \sum_{\alpha \in \Sigma_{Q}} \pm m_{\alpha} \alpha(H) \leq \rho_{Q}(H)
$$

for a suitable choice of signs, because $m_{\alpha}=m_{-\alpha}$ for $\alpha \in \Sigma_{A}=\Sigma_{Q} \cup-\Sigma_{Q}$. Now (i) follows easily.

The set of $F^{\prime}$-roots $\Sigma_{A^{\prime}}^{\prime}$ is the set of nonzero restrictions of elements of $\Sigma_{A}$ to $\mathfrak{a}^{\prime}$, and $m_{\alpha^{\prime}}$ is the sum of $m_{\alpha}$ over all $\alpha$ with $\left.\alpha\right|_{\mathfrak{a}^{\prime}}=\alpha^{\prime}$. Using the first formula from (i), we get the first assertion of (ii). Given $H \in \mathfrak{a}$ with image $H^{\prime} \in \mathfrak{a}^{\prime}$, we choose a parabolic $F^{\prime}$-subgroup $P^{\prime}$ with Levi component $M^{\prime}$ such that $H^{\prime} \in \overline{\mathfrak{a}_{P^{\prime}}^{\prime+}}$ and thus $\left|H^{\prime}\right|^{\prime}=\rho_{P^{\prime}}\left(H^{\prime}\right)$. There exists $P \in \mathcal{P}(A)$ such that $\mathfrak{a}_{P^{\prime}}^{+} \subset \overline{\mathfrak{a}_{P}^{+}}$, i.e., $P \subset P^{\prime}$. The value of $H^{\prime}$ on $\delta_{P^{\prime}} \in X^{\prime}\left(P^{\prime}\right) \cong X^{\prime}\left(M^{\prime}\right)$ equals the value of $H$ on $\left.\delta_{P^{\prime}}\right|_{M}$, namely

$$
\sum_{\substack{\left.\alpha \in \Sigma_{P} \\ \alpha\right|_{A^{\prime}} \neq 1}} m_{\alpha}|\alpha(H)| \leq 2|H| .
$$

Given special $F$-tori $A^{\prime} \subset A$ in $G$ and $P^{\prime} \in \mathcal{P}\left(A^{\prime}\right)$, we define $\mathfrak{a}_{P^{\prime}}^{+}$to be just $\mathfrak{a}_{P^{\prime}}^{+}$. Then $\mathfrak{a}$ is the disjoint union of the sets $\mathfrak{a}_{P^{\prime}}^{+}$where $P^{\prime}$ runs through all parabolic $F$-subgroups of $G$ containing the centraliser $M$ of $A$. Let $W_{A}$ denote the ( $F$-rational) Weyl group of $A$ in $G$ (i.e., the quotient of the normaliser by the centraliser of $A$ in $G(F))$. Then $\left.w \mathfrak{a}_{P^{\prime}}^{+}=\mathfrak{a}_{(w}^{+} P^{\prime}\right)$, where ${ }^{w} P^{\prime}:=\tilde{w} P^{\prime} \tilde{w}^{-1}$ for a representative $\tilde{w}$ of $w$. The Weyl group $W_{A}^{M^{\prime}}$ of $A$ in $M^{\prime}$ is the pointwise stabiliser of $A^{\prime}$ in $W_{A}$.

Lemma 2 Let $A^{\prime} \subset A$ be special F-tori, $w \in W_{A}$ and $P^{\prime} \in \mathcal{P}\left(A^{\prime}\right)$.

(i) If ${ }^{w} P^{\prime}=P^{\prime}$, then $w$ acts trivially on $\mathfrak{a}^{\prime}$.

(ii) The space of $w$-fixed vectors in $\mathfrak{a}$ is a special subspace, i.e., the real Lie algebra of a special F-torus $A^{\prime \prime}$. The centraliser $M^{\prime \prime}$ of $A^{\prime \prime}$ is the smallest Levi subgroup containing both $\tilde{w}$ and the centraliser $M$ of $A$.

(iii) In the situation of (ii), we have ${ }^{w} P^{\prime}=P^{\prime}$ iff $M^{\prime \prime} \subset P^{\prime}$.

(iv) If $P \in \mathcal{P}(A)$ and $P^{\prime}$ contains both $P$ and ${ }^{w} P$, then ${ }^{w} P^{\prime}=P^{\prime}$. 
Proof. (i) Since $P^{\prime}$ coincides with its own normaliser, we have $\tilde{w} \in P^{\prime}$. As $M^{\prime}$ is the unique Levi component of $P^{\prime}$ containing the centraliser $M$ of $A, \tilde{w}$ normalises $M^{\prime}$. It is well known that the normaliser of $M^{\prime}$ in $P^{\prime}$ is $M^{\prime}$.

(ii) Let $\mathfrak{a}^{\prime \prime}$ denote the space of $w$-fixed vectors in $\mathfrak{a}$. Pick $H \in \mathfrak{a}^{\prime \prime}$, let $P^{\prime}$ be the unique parabolic such that $H \in \mathfrak{a}_{P^{\prime}}^{+}$. Then $w P^{\prime}=P^{\prime}$, and by (i) the span $\mathfrak{a}^{\prime}$ of $\mathfrak{a}_{P^{\prime}}^{+}$is contained in $\mathfrak{a}^{\prime \prime}$. This shows that $\mathfrak{a}^{\prime \prime}$ is a union of special subspaces. Since there are only finitely many special subspaces in $\mathfrak{a}$ and since a proper subspace of $\mathfrak{a}^{\prime \prime}$ is a null set for the Lebesgue measure on $\mathfrak{a}^{\prime \prime}$, we see that $\mathfrak{a}^{\prime \prime}$ itself must be special.

(iii) If ${ }^{w} P^{\prime}=P^{\prime}$, then $\mathfrak{a}_{P^{\prime}}^{+} \subset \mathfrak{a}^{\prime \prime}$ by (i), and the converse is trivial.

(iv) In this situation, $P^{\prime}$ and ${ }^{w^{-1}} P^{\prime}$ are conjugate parabolic subgroups containing the parabolic subgroup $P$. It is well known that they must coincide.

Suppose we are given parabolic $F$-subgroups $P$ and $Q$ of $G$. If there exist parabolic $F$-subgroups contained in both $P$ and $Q$, then there is a greatest such parabolic, which we denote by $P \wedge Q$. In this case, of course, $P \wedge Q=P \cap Q$, because the latter is an algebraic $F$-subgroup containing a parabolic subgroup. On the other hand, the set of parabolic $F$-subgroups containing both $P$ and $Q$ is closed under intersection and non-empty, as $G$ belongs to it. Hence there is always a least such parabolic, which we denote by $P \vee Q$. Clearly, $\overline{\mathfrak{a}_{P \vee Q}^{+}}=\overline{\mathfrak{a}_{P}^{+}} \cap \overline{\mathfrak{a}_{Q}^{+}}$.

Finally, let us recall some notation connected with parabolic descent. If $P^{\prime}$ is a parabolic $F$-subgroup of $G$ with Levi $F$-component $M^{\prime}$, the assignment $P \mapsto$ $P \cap M^{\prime}$ defines a bijection of the set of parabolic $F$-subgroups of $G$ contained in $P^{\prime}$ onto the set of parabolic $F$-subgroups of $M^{\prime}$. We write $\Sigma_{P}^{P^{\prime}}, \Delta_{P}^{P^{\prime}}$ and $\rho_{P}^{P^{\prime}}$ for $\Sigma_{P \cap M^{\prime}}, \Delta_{P \cap M^{\prime}}$ and $\rho_{P \cap M^{\prime}}$, resp., which are (sets of) linear functionals on $\mathfrak{a}_{P}$ not depending on the choice of $M^{\prime}$. We denote by $\mathfrak{a}_{P}^{P^{\prime}}$ the kernel of the projection $\mathfrak{a}_{P} \rightarrow \mathfrak{a}_{P^{\prime}}$ dual to the restriction map $X\left(P^{\prime}\right) \rightarrow X(P)$, and we write $\left(\mathfrak{a}_{P}^{P^{\prime}}\right)^{+}$for the chamber corresponding to $P \cap M^{\prime}$. If $A^{\prime}$ is a maximal $F$-split torus in $P^{\prime}$ and $A \supset A^{\prime}$ a maximal $F$-split torus in $P$, we have identified $\mathfrak{a}_{P}$ with $\mathfrak{a}$ and $\mathfrak{a}_{P^{\prime}}$ with $\mathfrak{a}^{\prime}$, hence $\mathfrak{a}_{P^{\prime}} \subset \mathfrak{a}_{P}$. This yields a direct sum decomposition $\mathfrak{a}_{P}=\mathfrak{a}_{P}^{P^{\prime}} \oplus \mathfrak{a}_{P^{\prime}}$. The corresponding decomposition of elements $H \in \mathfrak{a}_{P}$ will be written as $H=H^{P^{\prime}}+H_{P^{\prime}}$.

Lemma 3 Let $P, Q \in \mathcal{P}(A)$ and set $P^{\prime}=P \vee Q$. Then there exists $c>0$ such that

$$
0<c \rho_{P}^{P^{\prime}}(H) \leq\left(\rho_{P}-\rho_{Q}\right)(H) \leq 2 \rho_{P}^{P^{\prime}}(H)
$$

for all $H \in \mathfrak{a}_{P}$ such that $H^{P^{\prime}} \in \overline{\left(\mathfrak{a}_{P}^{P^{\prime}}\right)^{+}}$.

Proof. Let $\Theta \subset \Delta_{P}$ be the set of primitive roots occurring in the decomposi- 
tion of elements of $\Sigma_{P} \cap \Sigma_{\bar{Q}}$, where $\Sigma_{\bar{Q}}=-\Sigma_{Q}$. Then

$$
\begin{aligned}
& \overline{\mathfrak{a}_{P}^{+}} \cap \overline{\mathfrak{a}_{Q}^{+}}=\left\{H \in \overline{\mathfrak{a}_{P}^{+}} \mid \alpha(H)=0 \quad \forall \alpha \in \Sigma_{P} \cap \Sigma_{\bar{Q}}\right\} \\
& =\left\{H \in \overline{\mathfrak{a}_{P}^{+}} \mid \alpha(H)=0 \quad \forall \alpha \in \Theta\right\}, \\
& \overline{\mathfrak{a}_{P^{\prime}}^{+}}=\left\{H \in \overline{\mathfrak{a}_{P}^{+}} \mid \alpha(H)=0 \quad \forall \alpha \in \Sigma_{P}^{P^{\prime}}\right\} .
\end{aligned}
$$

Since $\overline{\mathfrak{a}_{P^{\prime}}^{+}}=\overline{\mathfrak{a}_{P}^{+}} \cap \overline{\mathfrak{a}_{Q}^{+}}$, it follows that $\Theta=\Delta_{P}^{P^{\prime}}$. Moreover, $\Sigma_{P} \cap \Sigma_{\bar{Q}} \subset \Sigma_{P}^{P^{\prime}}$, and therefore

$$
\begin{gathered}
\rho_{P}-\rho_{Q}=\sum_{\alpha \in \Sigma_{P} \cap \Sigma_{\bar{Q}}} m_{\alpha} \alpha=\sum_{\beta \in \Delta_{P}^{P^{\prime}}} n_{\beta}^{\prime} \beta, \\
2 \rho_{P}^{P^{\prime}}=\sum_{\alpha \in \Sigma_{P}^{P^{\prime}}} m_{\alpha} \alpha=\sum_{\beta \in \Delta_{P}^{P^{\prime}}} n_{\beta} \beta
\end{gathered}
$$

whith positive $n_{\beta}, n_{\beta}^{\prime}$, because $\Sigma_{P} \cap \Sigma_{\bar{Q}}$ spans the dual of $\mathfrak{a}_{P}^{P^{\prime}}$.

\section{$3 \quad$ Inequality of descent}

Harish-Chandra has introduced the Schwartz space for $p=2$ only. It is an instance of his philosophy of descent that, roughly speaking, a Schwartz function $f$ on $G$ gives rise, for each parabolic $P$, to a Schwartz function $f^{P}$ on the Levi component of $P$. We shall extend the underlying inequality to the case $0<p \leq 2$.

Since the entire discussion is purely local, we change our notation throughout this section, denoting by $F$ a local field, by $G$ a connected reductive $F$-group and by $K$ a special maximal subgroup of $G(F)$. Hopefully, the notational simplification of dropping the subscript $v$ will outweigh the danger of confusing the reader. If $v$ is non-archimedean, we normalise the Haar measures on $G(F)$ and $K$ so that $K$ has measure one. Note that, for each parabolic $F$-subgroup $P$, the objects $X(P), \mathfrak{a}_{P}, \Sigma_{P}, \mathfrak{a}_{P}^{+}$and ${ }^{+} \mathfrak{a}_{P}$ are now defined with respect to the ground field $F$. We define the map $H_{P}: G(F) \rightarrow \mathfrak{a}_{P}$ by the same formula as in the global case. The modular character $d_{P}$ of $P(F)$ is given by $d_{P}(p)=\left|\delta_{P}(p)\right|=e^{2 \rho_{P}\left(H_{P}(p)\right)}$.

The definition of the space $\mathcal{C}^{p}(G(F))$ can be given in terms of the majorants

$$
\mu_{p, r}(g):=\Xi(g)^{2 / p}(1+\sigma(g))^{-r}, \quad g \in G(F),
$$

where we use the basic spherical function

$$
\Xi(g):=\int_{K} e^{\rho_{0}\left(H_{0}(k g)\right)} d k .
$$

Here the subscript 0 refers to some fixed minimal parabolic $F$-subgroup $P_{0}$, no matter which one, since all of them are $K$-conjugate. Remember that our $H_{0}$ is 
right $K$-invariant. The function $\sigma$ depends on the choice of a faithful $F$-rational representation $\eta$ of $G$ and a $K$-invariant norm on its representation space $V$. We set

$$
\sigma(g)=\log \max \left(\|\eta(g)\|,\left\|\eta\left(g^{-1}\right)\right\|\right),
$$

where $\|$. $\|$ denotes the Hilbert-Schmidt norm (resp. operator norm) on $\operatorname{End}_{F}(V)$ if $F$ is archimedean (resp. nonarchimedean).

Note that $\Xi(g)$ equals $\left(h_{0}, \pi_{0}(g) h_{0}\right)$, the matrix coefficient for a $K$-fixed unit vector $h_{0}$ of the representation $\pi_{0}$ unitarily induced from the trivial representation of $P_{0}$. If $h_{0}^{\prime}$ is fixed under a different special maximal compact subgroup $K^{\prime}$, then $h_{0}^{\prime}=\pi_{0}(f) h_{0}$ for some smooth compactly supported function $f$ on $G(F)$ by [23], Lemma 1.11.3, hence the resulting spherical function is $\Xi^{\prime}=f * \Xi * f^{*}$. In the archimedean case we have $\Xi^{\prime}(g)=\Xi\left(g_{0}^{-1} g g_{0}\right)$ for some $g_{0}$ by the conjugacy of maximal compact subgroups. If $\mu_{p, r}^{\prime}$ comes from $K^{\prime}$ and a different choice of $\eta$ and $\|$.$\| , then it follows from [25], Prop. 8.3.7.2 / [23], Lemma 4.2.3 and the$ remarks on p. 150, that for every $r>0$ there exist $C>0, r^{\prime}>0$ such that $\mu_{p, r} \leq C \mu_{p, r^{\prime}}^{\prime}$. Moreover, $\mu_{p, r} \in L^{p}(G(F))$ for large $r$ by [25], Prop. 8.3.7.5 / [23], Lemma 4.2.5. More precisely, if we fix a norm $\|$. $\|$ on $\mathfrak{a}_{G}$, there exist $C$ and $q>0$ such that

$$
\int_{G(F)^{1}} \mu_{p, r}\left(g_{0} g\right)^{p} d g \leq C\left(1+\left\|H_{G}\left(g_{0}\right)\right\|\right)^{-q r} .
$$

Let $A_{0}$ be a maximal $F$-split torus of $G$ whose apartment in the Bruhat-Tits building of $G$ contains the vertex fixed by $K$. In the archimedean case (cf. [22], section 5), this means that the Lie algebras of $K$ and $A_{0}(F)$ are orthogonal with respect to the Killing form. If $A$ is a special subgroup of $A_{0}$ and $M$ the centraliser of $A$, then $K_{M}:=K \cap M(F)$ is a special maximal subgroup of $M(F)$, and the elements of the Weyl group $W_{A}$ have representatives in $K$. We may assume in addition that $A_{0} \subset P_{0}$ (by changing $P_{0}$ or $A_{0}$, because minimal parabolic $F$ subgroups are $K$-conjugate). The inclusion map of the centraliser $M_{0}$ of $A_{0}$ into $G$ yields a bijection

$$
K_{M_{0}} \backslash M_{0}(F) / K_{M_{0}} \rightarrow K \backslash G(F) / K
$$

Therefore $\Xi$ and $\sigma$ are determined by their restrictions to

$$
M_{0}(F)^{+}:=\left\{m \in M_{0}(F) \mid H_{0}(m) \in \overline{\mathfrak{a}_{P_{0}}^{+}}\right\} .
$$

There are positive constants $C_{1}, C_{2}$ and $r_{0}$ such that

$$
C_{1} \leq d_{P_{0}}(m)^{1 / 2} \Xi(m) \leq C_{2}(1+\sigma(m))^{r_{0}}
$$

for $m \in M_{0}(F)^{+}$by [25], Prop. 8.3.7.3 and Th. 8.3.7.4 / [23], Th. 4.2.1. Thus, in the definition of $\mathcal{C}^{p}(G(F))$, we could have replaced $\Xi$ by the $K$-biinvariant function whose restriction to $M_{0}(F)$ is $e^{-\left|H_{0}(m)\right|}$ in the notation of Lemma 1 . 
Lemma 4 Let $P$ be a parabolic F-subgroup with unipotent radical $N$ and Levi component $M$ containing $M_{0}$. Given $0<p \leq 2, s>0$ and $r>s+\left(\frac{2}{p}+1\right) r_{0}$, there exists $C>0$ such that, for each $m \in M(F)$,

$$
d_{P}(m)^{1 / 2} \int_{N(F)} \mu_{p, r}(m n) d n \leq C \mu_{p, s}^{M}(m) e^{-\left(\frac{2}{p}-1\right)\left|H_{M}(m)\right|} .
$$

Here $\mu_{p, r}^{M}$ is defined using the basic $K_{M}$-spherical function on $M(F)$ and the restriction of $\sigma$ to $M(F)$.

For $p=2$, this is just [11], Lemma $21 /$ [13], Cor. 2 of Th. 25 (cf. also [25], Th. 8.5.2.1 / [23], Th. 4.3.20). For the proof in the general case, we need the following convexity theorem: If $m_{0} \in M_{0}(F)$ and $k \in K$, then $H_{0}\left(k m_{0}\right)$ lies in the convex hull of the $W_{A_{0}}$-orbit of $H_{0}\left(m_{0}\right)$. In the archimedean case, this has been proved in [14], while a proof for the non-archimedean case has been sketched in [24]. (We will not need Kostant's full convexity theorem, which describes $\left\{H_{0}\left(k m_{0}\right) \mid k \in K\right\}$ completely, nor its full analogue in the non-archimedean case, for which the details of proof in [24] are scarce.) Thus,

$$
H_{0}\left(k m_{0}\right)=\sum_{w \in W_{A_{0}}} a_{w} w H_{0}\left(m_{0}\right) \quad \text { with } \quad a_{w}>0, \quad \sum_{w \in W_{A_{0}}} a_{w}=1,
$$

and hence

$$
\left|H_{0}\left(k m_{0}\right)\right| \leq \sum_{w \in W_{A_{0}}} a_{w}\left|w H_{0}\left(m_{0}\right)\right|=\left|H_{0}\left(m_{0}\right)\right|
$$

due to Lemma 1 and the $W_{A_{0}}$-invariance of $\mid$.|. If $m_{0} \in M_{0}(F)^{+}$, then the righthand side equals $\rho_{0}\left(H_{0}\left(m_{0}\right)\right)$. Combined with $(2)$ and the $K$-biinvariance of $\Xi$ and $\sigma$, this implies that

$$
\Xi(g) \leq C_{2} e^{-\left|H_{0}(g)\right|}(1+\sigma(g))^{r_{0}}
$$

for all $g \in G(F)$. In particular, for $m_{0} \in M_{0}(F)$ and $n_{0} \in N_{0}(F)$ we get

$$
\Xi\left(m_{0} n_{0}\right) \leq=C_{2} e^{-\left|H_{0}\left(m_{0}\right)\right|}\left(1+\sigma\left(m_{0} n_{0}\right)\right)^{r_{0}} .
$$

The same is true for nonminimal parabolics, too: Let $m \in M(F), n \in N(F)$. Then $m=k_{1} m_{0} k_{2}$ with $k_{1}, k_{2} \in K_{M}$ and $m_{0} \in M_{0}(F)$, hence $\left|H_{0}\left(m_{0}\right)\right| \geq$ $\left|H_{M}\left(m_{0}\right)\right|=\left|H_{M}(m)\right|$ by Lemma 1(ii). Setting $n_{0}=k_{2} n k_{2}^{-1}$, we get

$$
\Xi(m n)=\Xi\left(m_{0} n_{0}\right) \leq C_{2} e^{-\left|H_{M}(m)\right|}(1+\sigma(m n))^{r_{0}} .
$$

This shows that, for $0<p \leq 2$,

$$
\mu_{p, r+r_{2}}(n m) \leq C_{2}^{2 / p} \mu_{2, r}(n m) e^{-\left(\frac{2}{p}-1\right)\left|H_{M}(m)\right|}
$$

with $r_{2}=\left(\frac{2}{p}-1\right) r_{0}$, and the Lemma follows from the special case $p=2$ when we integrate over $n \in N(F)$. 


\section{Sums over Bruhat cells}

We return to the setting of section 1, thus denoting by $F$ a global field again. The results of the previous section are applicable to the local field $F_{v}$ for each place $v$ of $F$. The majorants on $G\left(F_{v}\right)$ which were denoted by $\mu_{p, r}$ should now be written as $\mu_{v, p, r}$. If $S$ is a finite set of places, then functions in $\mathcal{C}^{p}\left(G\left(F_{S}\right)^{1}\right)$ and their invariant derivatives are bounded by

$$
\mu_{S, p, r}(g):=\prod_{v \in S} \mu_{v, p, r}\left(g_{v}\right)
$$

As in section 1 , we extend this to a $K^{S}$-invariant function on $G(\mathbb{A})$ vanishing outside $G\left(F_{S}\right) K^{S}$.

Now we adapt Lemma 4 to this global setting. Let $A$ be a special $F$-torus with centraliser $M$, and let $P \in \mathcal{P}(A)$ with unipotent radical $N$. For each place $v$ of $F$, let $A_{v}$ be the greatest $F_{v}$-split torus in the centre of $M$. As explained in section 3 , the dependence of $\mu_{v, p, r}$ on $K_{v}$ is insignificant. Hence we may assume that $K_{v}$ is in good relative position to $A_{v}$ for each $v$. By Lemma 1(ii),

$$
\left|H_{M}\left(m_{v}\right)\right| \leq\left|H_{M, v}\left(m_{v}\right)\right|_{v} \quad \text { for } m_{v} \in M\left(F_{v}\right),
$$

where the map $H_{M, v}$ and the seminorm $|.|_{v}$ on $\mathfrak{a}_{v}$ are defined with respect to the ground field $F_{v}$. Lemma 4 now yields, after taking the product over $v \in S$,

$$
d_{P}(m)^{1 / 2} \int_{N\left(F_{S}\right)} \mu_{S, p, r}(m n) d n \leq C_{1} \mu_{S, p, s}^{M}(m) e^{-\left(\frac{2}{p}-1\right)\left|H_{M}(m)\right|}
$$

for all $m \in M\left(F_{S}\right)$ and $r>s+\left(\frac{2}{p}+1\right) r_{0}$, where $d_{P}(m)=\left|\delta_{P}(m)\right|$ with $|$. denoting the idele norm. By our assumption on $K_{v}$ we have

$$
K_{v} \cap P\left(F_{v}\right)=\left(K_{v} \cap M\left(F_{v}\right)\right)\left(K_{v} \cap N\left(F_{v}\right)\right) .
$$

Thus, if we normalise the measure on $N\left(\mathbb{A}^{S}\right)$ so that $\operatorname{vol}\left(K^{S} \cap N\left(\mathbb{A}^{S}\right)\right)=1$, we get

$$
d_{P}(m)^{1 / 2} \int_{N(\mathbb{A})} \mu_{S, p, r}(m n) d n \leq C_{1} \mu_{S, p, s}^{M}(m) e^{-\left(\frac{2}{p}-1\right)\left|H_{M}(m)\right|}
$$

for all $m \in M(\mathbb{A})$, which is the global analogue of Lemma 4. Similarly, the estimate (1) implies the existence of constants $C_{2}, q>0$ such that, for large $r$,

$$
\int_{G(\mathbb{A})^{1}} \mu_{S, p, r}\left(g_{0} g\right)^{p} d g \leq C_{2}\left(1+\left\|H_{G}\left(g_{0}\right)\right\|\right)^{-q r} .
$$

Here we have split the integral and evaluated the one over $G\left(F_{S}\right)^{1} / \prod_{v \in S} G\left(F_{v}\right)^{1}$ using the fact that $\prod_{v \in S} H_{G, v}$ embeds that group into the vector space $\prod_{v \in S} \mathfrak{a}_{G, v}$. 
Now we are going to state our main technical estimate, which has been inspired by Lemma 11.4 of [21]. We fix a minimal parabolic $F$-subgroup $P_{0}$ of $G$ and its Levi decomposition $P_{0}=M_{0} N_{0}$ defined over $F$ and denote by $A_{0}$ the greatest $F$-split torus in the centre of $M_{0}$. As $F$ is now a global field, this notation differs from that used in the preceding section. The set $G(F)$ is the disjoint union of the Bruhat cells

$$
G_{w}(F):=P_{0}(F) \tilde{w} P_{0}(F),
$$

where $w$ runs through the Weyl group $W_{0}$ of $A_{0}$ in $G$ and $\tilde{w} \in G(F)$ is a representative of $w$. For $x, y \in G(\mathbb{A})$, we set

$$
\kappa_{S, p, r}^{w}(x, y)=\sum_{\gamma \in G_{w}(F)} \mu_{S, p, r}\left(x^{-1} \gamma y\right) .
$$

Theorem 4 Let $P_{0}, S$ and $w$ be as above, and set $P=P_{0} \vee^{w} P_{0}$ in the notation introduced in section 2. Then, for every $0<p<1$ and large $r$, one can find $C>0$ such that, for all $x, y \in G(\mathbb{A})^{1}$,

$$
\kappa_{S, p, r}^{w}(x, y) \leq C e^{\left|H_{0}(x)\right|+\left|H_{0}(y)\right|} e^{-\left(\frac{2}{p}-1\right)\left(\left|H_{P}(x)-H_{P}(y)\right|-w \rho_{0}^{P}\left(H_{0}(x)\right)+\rho_{0}^{P}\left(H_{0}(y)\right)\right)} .
$$

Corollary 1 In the situation of the theorem, there exists $c>0$ with the following property. For each truncation parameter $T \in \mathfrak{a}_{0}, 0<p<1$ and large $r$ one can find $C>0$ such that

$$
\kappa_{S, p, r}^{w}(x, y) \leq C e^{-c\left(\frac{2}{p}-1\right)\left|H_{0}(x)-w H_{0}(y)\right|+\left|H_{0}(x)\right|+\left|H_{0}(y)\right|}
$$

for all $x, y \in G(\mathbb{A})^{1}$ satisfying $\left(H_{0}(x)-T\right)^{P},\left(H_{0}(y)-T\right)^{P} \in \overline{\left(\mathfrak{a}_{0}^{P}\right)^{+}}$.

Let us deduce the corollary from the bound provided in the theorem, which we symmetrise as

$$
\kappa_{S, p, r}^{w}(x, y)=\kappa_{S, p, r}^{w^{-1}}(y, x)=\left(\kappa_{S, p, r}^{w}(x, y) \kappa_{S, p, r}^{w^{-1}}(y, x)\right)^{1 / 2}
$$

to obtain

$$
\begin{aligned}
& \log \left(\kappa_{S, p, r}^{w}(x, y)\right) \leq c_{1}+\left|H_{0}(x)\right|+\left|H_{0}(y)\right| \\
& -\left(\frac{2}{p}-1\right)\left(\left|H_{P}(x)-H_{P}(y)\right|+\frac{1}{2}\left(\rho_{0}^{P}-w \rho_{0}^{P}\right)\left(H_{0}(x)\right)+\frac{1}{2}\left(\rho_{0}^{P}-w^{-1} \rho_{0}^{P}\right)\left(H_{0}(y)\right)\right)
\end{aligned}
$$

By Lemma 2(iv) we have $\tilde{w} \in P(F)$, hence $H_{P}(y)=w H_{P}(y)$ and $\rho_{0}^{P}-w \rho_{0}^{P}=$ $\rho_{0}-\rho_{P}-w \rho_{0}+w \rho_{P}=\rho_{0}-w \rho_{0}$. Now Lemma 3 implies

$$
\frac{1}{2}\left(\rho_{0}^{P}-w \rho_{0}^{P}\right)\left(H_{0}(x)-T\right) \geq c_{2} \rho_{0}^{P}\left(H_{0}(x)-T\right) \geq c_{3}\left|\left(H_{0}(x)-T\right)^{P}\right|,
$$

and similarly

$$
\frac{1}{2}\left(\rho_{0}^{P}-w^{-1} \rho_{0}^{P}\right)\left(H_{0}(y)-T\right) \geq c_{2} \rho_{0}^{P}\left(H_{0}(y)-T\right) \geq c_{3}\left|w\left(H_{0}(y)-T\right)^{P}\right|
$$


due to the $W_{0}$-invariance of $|$.$| . The triangle inequality implies$

$$
\left|H_{P}(x)-w H_{P}(y)\right|+c_{3}\left|H_{0}(x)^{P}\right|+c_{3}\left|w H_{0}(y)^{P}\right| \geq c_{3}\left|H_{0}(x)-w H_{0}(y)\right|
$$

and allows us to get rid of the $T$-shifts at the cost of modifying $C$.

This section and the next one are devoted to the proof of Theorem 4. At first, we derive an estimate on the averaged function

$$
\bar{\kappa}_{S, t, r}^{w}(x, y):=\int_{N_{0}(F) \backslash N_{0}(\mathbb{A})} \int_{P_{0}(F) \backslash P_{0}(\mathbb{A})^{1}} \kappa_{S, t, r}^{w}(n x, p y) d p d n .
$$

Lemma 5 For $0<t<1$ and large $r$ there is $C$ such that, for $x, y \in G(\mathbb{A})^{1}$,

$$
\bar{\kappa}_{S, t, r}^{w}(x, y) \leq C e^{\left(\rho_{0}+\left(\frac{2}{t}-1\right) w \rho_{0}\right)\left(H_{0}(x)\right)+\left(2-\frac{2}{t}\right) \rho_{0}\left(H_{0}(y)\right)} .
$$

Proof. Since both sides are right $K$-invariant in both arguments, we may assume that $x, y \in P_{0}(\mathbb{A})$, in which case

$$
\bar{\kappa}_{S, t, r}^{w}(x, y)=d_{0}(y) \int_{N_{0}(F) \backslash N_{0}(\mathbb{A})} \sum_{\gamma \in P_{0}(F) \backslash G_{w}(F)} \int_{P_{0}(\mathbb{A})^{1}} \mu_{S, t, r}\left((\gamma n x)^{-1} y p\right) d p d n .
$$

Equation (3) implies that, for each $z \in G(\mathbb{A})$ and $r>s+\left(\frac{2}{t}+1\right) r_{0}$,

$$
\int_{P_{0}(\mathbb{A})^{1}} \mu_{S, t, r}\left(z^{-1} p\right) d p \leq C_{1} e^{\frac{2}{t} \rho_{0}\left(H_{0}(z)\right)} \int_{M_{0}(\mathbb{A})^{1}} \mu_{S, t, s}^{M_{0}}\left(m_{0}^{-1} m\right) d m,
$$

where $m_{0} \in M(\mathbb{A})$ is such that $z \in N_{0}(\mathbb{A}) m_{0} K$. For sufficiently large $r$ we can choose $s$ such that (4) applies to the integral on the right-hand side. Now we set $z=y^{-1} \gamma n x$, sum over $\gamma$ and integrate over $n$ to get

$$
\bar{\kappa}_{S, t, r}^{w}(x, y) \leq C_{2} d_{0}(y)^{1-\frac{1}{t}} \int_{N_{0}(F) \backslash N_{0}(\mathbb{A})} \sum_{\gamma \in P_{0}(F) \backslash G_{w}(F)} e^{\frac{2}{t} \rho_{0}\left(H_{0}(\gamma n x)\right)} d n .
$$

The integral on the right-hand side is the constant term of a partial Eisenstein series, hence of the form

$$
c\left(w,\left(\frac{2}{t}-1\right) \rho_{0}\right) e^{\left(\rho_{0}+\left(\frac{2}{t}-1\right) w \rho_{0}\right)\left(H_{0}(x)\right)},
$$

where $c(w, \lambda)$ is holomorphic on the set of all $\lambda \in \mathfrak{a}_{P, \mathbb{C}}$ such that $\operatorname{Re} \lambda-\rho_{P}$ belongs to the dual cone $\left(\mathfrak{a}_{0}^{*}\right)^{+}$of ${ }^{+} \mathfrak{a}_{0}$ (see $\left.[10], \S 3\right)$.

We must strengthen Lemma 5 because it does not exhibit the decay of $\bar{\kappa}_{S, t, r}^{w}$ we need.

Lemma 6 For $0<t<1$ and large $r$ there exists $C$ such that, for $x, y \in G(\mathbb{A})^{1}$,

$$
\bar{\kappa}_{S, t, r}^{w}(x, y)=C e^{\rho_{0}\left(H_{0}(x)+H_{0}(y)\right)} e^{-\left(\frac{2}{t}-1\right)\left(\left|H_{P}(x)-w H_{Q}(y)\right|-w \rho_{0}^{P}\left(H_{0}(x)\right)+\rho_{0}^{P}\left(H_{0}(y)\right)\right)} .
$$


Proof. Let $P=M N$ be the Levi decomposition with $M \supset M_{0}$. Then $P_{0}^{\prime}:=P_{0} \cap M$ is a minimal parabolic $F$-subgroup of $M$ with Levi component $M_{0}$ and unipotent radical $N_{0}^{\prime}:=N_{0} \cap M$. Since $\gamma^{-1} N \gamma \subset N_{0}$ for $\gamma \in G_{w}(F)$, we see that

$$
\int_{P_{0}(\mathbb{A})^{1}} \mu_{S, t, r}\left(x^{-1} \gamma p y\right) d p
$$

is left $N(\mathbb{A})$-invariant as a function of $x$. In view of (5) we may, in the definition of $\bar{\kappa}_{S, t, r}^{w}$, replace the integral over $N_{0}(F) \backslash N_{0}(\mathbb{A})$ by $\operatorname{vol}(N(F) \backslash N(\mathbb{A}))$ times an integral over $N_{0}^{\prime}(F) \backslash N_{0}^{\prime}(\mathbb{A})$.

By Lemma 2(iv), we have $w \in W_{0}^{M}, \tilde{w} \in M(F)$, and therefore

$$
G_{w}=P_{0}^{\prime} N \tilde{w} P_{0}^{\prime} N=P_{0}^{\prime} \tilde{w} P_{0}^{\prime} N=M_{w} N .
$$

Consequently,

$$
\bar{\kappa}_{S, t, r}^{w}(x, y)=\int_{N_{0}^{\prime}(F) \backslash N_{0}^{\prime}(\mathbb{A})} \sum_{\gamma \in M_{w}(F) / P_{0}^{\prime}(F)} \int_{P_{0}(\mathbb{A})^{1}} \mu_{S, t, r}\left(x^{-1} n^{-1} \gamma p y\right) d p d n^{\prime} .
$$

We split the inner integral according to $P_{0}=P_{0}^{\prime} N$ and deduce from the inequality of descent (3) that it does not exceed

$$
C_{1} d_{P}(x y)^{1 / 2} e^{-\left(\frac{2}{t}-1\right)\left|H_{P}\left(x^{-1} y\right)\right|} \int_{P_{0}^{\prime}(\mathbb{A})^{1}} \mu_{S, t, s}^{M}\left(x^{\prime-1} n^{-1} \gamma p^{\prime} y^{\prime}\right) d p^{\prime},
$$

where $x^{\prime}, y^{\prime}$ are the projections of $x, y$ on $M(\mathbb{A})$ along $N(\mathbb{A})$. Note that we have picked an additional factor $d_{P}(y)$ by a substitution in the integral over $N(\mathbb{A})$. Applying now Lemma 5 with $\left(G, P_{0}\right)$ replaced by $\left(M, P_{0}^{\prime}\right)$, we obtain

$$
\begin{aligned}
\bar{\kappa}_{S, t, r}^{w}(x, y) \leq C_{2} e^{\rho_{P}\left(H_{P}(x)+H_{P}(y)\right)} e^{-\left(\frac{2}{t}-1\right)\left|H_{P}\left(x^{-1} y\right)\right|} & \\
& \times e^{\left(\rho_{0}^{\prime}+\left(\frac{2}{t}-1\right) w \rho_{0}^{\prime}\right)\left(H_{0}^{\prime}\left(x^{\prime}\right)\right)+\left(2-\frac{2}{t}\right) \rho_{0}^{\prime}\left(H_{0}^{\prime}\left(y^{\prime}\right)\right)} .
\end{aligned}
$$

We can rewrite the right-hand side as claimed in the lemma by using the facts that $\rho_{0}^{\prime}\left(H_{0}^{\prime}\left(x^{\prime}\right)\right)=\rho_{0}^{P}\left(H_{0}(x)\right)$ and that $w$ acts trivially on $\mathfrak{a}$.

\section{$5 \quad$ Bounding sums by integrals}

To finish the proof of Theorem 4, we still have to bound $\kappa_{S, t, r}^{w}$ in terms of $\bar{\kappa}_{S, t, r}^{w}$. The usual way to estimate a sum over a lattice is to replace the value of the function at each lattice point by an integral over a small neighbourhood. In our situation, this leads to the problem of counting the number of terms in a sum like the one defining the kernel $K(x, x)$ for compactly supported $f$. There is a standard estimate on $K(x, y)$ not depending on $y$ (cf. [19], I.2.4), but the following lemma will enable us to obtain a sharper bound in the case at hand. 
Lemma 7 Let $P_{0}$ be a minimal parabolic $F$-subgroup and $\Omega$ a compact subset of $P_{0}(\mathbb{A})^{1}$. Then there exists $C>0$ such that, for all $x \in P_{0}(\mathbb{A})$ and $p_{1}, p_{2} \in P_{0}(\mathbb{A})^{1}$

$$
\#\left({ }^{x} \Omega \cap p_{1} P_{0}(F) p_{2}\right)<C d_{0}(x)^{1 / 2} e^{\left|H_{0}(x)\right|},
$$

where ${ }^{x} \Omega:=x \Omega x^{-1}$.

Proof. We assert that the set

$$
B=\left\{p^{-1} \gamma p \mid p \in P_{0}(\mathbb{A})^{1}, \gamma \in P_{0}(F) \backslash\{1\}\right\}
$$

is closed in $P_{0}(\mathbb{A})$. Indeed, consider the maps

$$
P_{0}(\mathbb{A}) \stackrel{\varphi}{\longleftarrow} P_{0}(\mathbb{A})^{1} \times P_{0}(\mathbb{A}) \stackrel{\pi_{1}}{\longrightarrow}\left(P_{0}(F) \backslash P_{0}(\mathbb{A})^{1}\right) \times P_{0}(\mathbb{A}) \stackrel{\pi_{2}}{\longrightarrow} P_{0}(\mathbb{A})
$$

where $\pi_{1}, \pi_{2}$ are the natural projections and $\varphi(p, q)=p q p^{-1}$. Then the set $\pi_{1}^{-1}\left(\pi_{2}^{-1}(B)\right)=\varphi^{-1}\left(P_{0}(F) \backslash\{1\}\right)$ is closed due to the discreteness of $P_{0}(F)$, hence $\pi_{2}^{-1}(B)$ is closed and, in view of the compactness of $P_{0}(F) \backslash P_{0}(\mathbb{A})^{1}, B$ is closed.

This allows us to choose a compact neighbourhood $\omega$ of 1 in $P_{0}(\mathbb{A})^{1}$ such that $\omega \omega^{-1} \cap B=\emptyset$. Then the sets $p_{1} \gamma p_{2} \omega$ with $\gamma \in P_{0}(F)$ are disjoint, and

$$
\#\left({ }^{x} \Omega \cap p_{1} P_{0}(F) p_{2}\right) \operatorname{vol}(\omega)=\operatorname{vol}\left(\left({ }^{x} \Omega \cap p_{1} P_{0}(F) p_{2}\right) \omega\right) \leq \operatorname{vol}\left({ }^{x} \Omega \omega\right),
$$

where vol refers to the Haar measure of $P_{0}(\mathbb{A})^{1}$.

Let $A_{0}$ be as above, and fix a valuation $v_{0}$ of $F$ such that the norm functions on $\mathbb{A}$ and $F_{v_{0}}$ have the same range. If $Q_{0} \in \mathcal{P}\left(A_{0}\right)$ with unipotent radical $U_{0}$, say, then any compact subset $\omega^{\prime} \subset U_{0}(\mathbb{A})$ can be enlarged to a compact subset $\omega^{\prime \prime} \in U_{0}(\mathbb{A})$ such that ${ }^{a} \omega^{\prime \prime} \subset \omega^{\prime \prime}$ for all $a \in A_{0}\left(F_{v_{0}}\right)$ satisfying $H_{0}(a) \in \overline{\mathfrak{a}_{0, Q_{0}}^{-}}=$ $\overline{\mathfrak{a}_{0, \bar{Q}_{0}}^{+}}$. To see this, recall from [5], $\S 9.12$, that there are $F$-morphisms $\phi_{\alpha}$ of vector groups $V_{\alpha}$ into $U_{0}$ such that

$$
\phi_{\alpha}(\alpha(a) v)=a \phi_{\alpha}(v) a^{-1}
$$

for $a \in A_{0}$ and $v \in V_{\alpha}$ and such that the product map

$$
\prod_{\alpha \in \Sigma_{Q_{0}}} V_{\alpha} \rightarrow U_{0}
$$

(any fixed order of factors) is an isomorphism of $F$-manifolds. Given any compact subset $\tilde{\omega}^{\prime} \subset \prod_{\alpha \in \Sigma_{Q_{0}}} V_{\alpha}(\mathbb{A})$, the image $\tilde{\omega}^{\prime \prime}$ of $\tilde{\omega}^{\prime} \times\left\{c \in F_{v_{0}}|| c \mid \leq 1\right\}^{\Sigma_{Q_{0}}}$ under componentwise multiplication is compact, too.

Now we can deduce the lemma from the inequality (6). By changing $p_{1}$ and $p_{2}$, we may assume that $x=a \in A_{0}\left(F_{v_{0}}\right)$. Now we choose $Q_{0}$ such that $H_{0}(a) \in \overline{\mathfrak{a}_{0, Q_{0}}^{+}}$and set $P_{+}=M_{0}\left(N_{0} \cap U_{0}\right), P_{-}=N_{0} \cap \bar{U}_{0}$. Then the product map $P_{+}(\mathbb{A})^{1} \times P_{-}(\mathbb{A}) \rightarrow P_{0}(\mathbb{A})^{1}$ is an isomorphism of measure spaces. As we have just 
seen, we can produce suitable compact sets $\Omega_{ \pm} \subset P_{ \pm}(\mathbb{A})^{1}$ with $\Omega \subset \Omega_{+} \Omega_{-}$and further compact sets $\Omega_{ \pm}^{\prime} \subset P_{ \pm}(\mathbb{A})^{1}$ with $\Omega_{-} \omega \subset \Omega_{+}^{\prime} \Omega_{-}^{\prime}$ such that

$$
{ }^{a} \Omega \omega \subset{ }^{a} \Omega_{+} \Omega_{-} \omega \subset{ }^{a} \Omega_{+} \Omega_{+}^{\prime} \Omega_{-}^{\prime} \subset{ }^{a}\left(\Omega_{+} \Omega_{+}^{\prime}\right) \Omega_{-}^{\prime},
$$

and we get

$$
\operatorname{vol}\left({ }^{a} \Omega \omega\right)=d_{+}(a) \operatorname{vol}\left(\Omega_{+} \Omega_{+}^{\prime} \Omega_{-}^{\prime}\right),
$$

where $d_{+}$is the modular character of $P_{+}(\mathbb{A})$, which satisfies

$$
d_{+}(a)=\prod_{\alpha \in \Sigma_{P_{0}} \cap \Sigma_{Q_{0}}}|\alpha(a)|^{m_{\alpha}}=d_{P_{0}}(a)^{1 / 2} d_{Q_{0}}(a)^{1 / 2}=d_{P_{0}}(a)^{1 / 2} e^{\left|H_{0}(a)\right|}
$$

for the given $a$. Since there are only finitely many choices of $Q_{0}$, one $C$ suits all of them.

Corollary 2 If $f$ is a non-negative function on $P_{0}(\mathbb{A})$, then

$$
\sum_{\gamma \in P_{0}(F)} \int_{\Omega} f(\gamma x p) d p \leq C d_{0}(x)^{-1 / 2} e^{\left|H_{0}(x)\right|} \int_{P_{0}(\mathbb{A})^{1}} f(p x) d p
$$

for all $x \in P_{0}(\mathbb{A})$. If, moreover, $\Omega$ is contained in $N_{0}(\mathbb{A})$, then

$$
\sum_{\gamma \in N_{0}(F)} \int_{\Omega} f(\gamma x n) d n \leq C d_{0}(x)^{-1 / 2} e^{\left|H_{0}(x)\right|} \int_{N_{0}(\mathbb{A})} f(n x) d n
$$

for all $x \in P_{0}(\mathbb{A})$, where dn denotes a Haar measure on $N_{0}(\mathbb{A})$.

Indeed, the left-hand side of the first inequality equals

$$
d_{0}(x)^{-1} \sum_{\gamma \in P_{0}(F)} \int_{x_{\Omega}} f(\gamma p x) d p=d_{0}(x)^{-1} \int_{P_{0}(\mathbb{A})^{1}} f\left(p_{2} x\right) \sum_{\gamma \in P_{0}(F)} \chi_{\gamma, x}\left(p_{2}\right) d p_{2},
$$

where $\chi_{\gamma, x}$ is the characteristic function of $\gamma \cdot{ }^{x} \Omega$. Now one can bound the sum with the aid of the lemma. The second inequality is proved in the same way.

Lemma 8 Let $P_{0}, S$ and $w$ be as in the preceding section. Given $t>0$ and $r>0$, there exists $C>0$ such that

$$
\kappa_{S, t, r}^{w}(x, y) \leq C e^{\left|H_{0}(x)\right|-\rho_{0}\left(H_{0}(x)\right)} e^{\left|H_{0}(y)\right|-\rho_{0}\left(H_{0}(y)\right)} \bar{\kappa}_{S, t, r}^{w}(x, y)
$$

for all $x, y \in G(\mathbb{A})$. 
Proof. As in Lemma 5, it suffices to consider $x, y \in P_{0}(\mathbb{A})$. Given compact sets $\Omega, \Omega^{\prime} \subset G\left(F_{S}\right) K^{S}$, one easily deduces from [25], Prop. 8.3.7.2 / [23], Lemma 4.2.3 that there exists $C_{1}>0$ such that

$$
\mu_{S, t, r}(g) \leq C_{1} \mu_{S, t, r}\left(n^{-1} g p\right)
$$

for all $g \in G(\mathbb{A}), p \in \Omega$ and $n \in \Omega^{\prime}$. We may assume that $\Omega \subset P_{0}(\mathbb{A})^{1}, \Omega^{\prime} \subset N_{0}(\mathbb{A})$ and that each set has positive Haar measure in the pertinent group. Setting $g=x^{-1} \gamma y$ with $\gamma \in G(F)$ and integrating, we get

$$
\mu_{S, t, r}\left(x^{-1} \gamma y\right) \leq C_{2} \int_{\Omega^{\prime}} \int_{\Omega} \mu_{S, t, r}\left(n^{-1} x^{-1} \gamma y p\right) d p d n .
$$

Now we sum over $\gamma \in G_{w}(F)$ and apply the corollary of Lemma 7 to see that $\kappa_{S, t, r}^{w}(x, y)$ is bounded by

$$
C_{3} \int_{\Omega^{\prime}} \sum_{\gamma \in G_{w}(F) / P_{0}(F)} d_{0}(y)^{-1 / 2} e^{\left|H_{0}(y)\right|} \int_{P_{0}(\mathbb{A})^{1}} \mu_{S, t, r}\left(n^{-1} x^{-1} \gamma p y\right) d p d n,
$$

which can be written as

$$
C_{3} d_{0}(y)^{-1 / 2} e^{\left|H_{0}(y)\right|} \int_{P_{0}(F) \backslash P_{0}(\mathbb{A})^{1}} \sum_{\gamma \in G_{w}(F)} \int_{\Omega^{\prime}} \mu_{S, t, r}\left((x n)^{-1} \gamma p y\right) d n d p .
$$

Another application of the corollary, this time to the integral over $n$, completes the proof.

Theorem 4 clearly follows from Lemmas 6 and 8 .

\section{Alternating sums of constant terms}

Recall that the constant term of a locally integrable function $\phi$ on $N(F) \backslash G(\mathbb{A})$, where $N$ is the unipotent radical of a parabolic $F$-subgroup $P$, is defined as

$$
\Pi^{P} \phi(x)=\int_{N(F) \backslash N(\mathbb{A})} \phi(n x) d n,
$$

where the $N(\mathbb{A})$-invariant measure $d n$ on $N(F) \backslash N(\mathbb{A})$ is normalised to have total mass one. We will need a basic inequlity concerning alternating sums

$$
\Pi_{P}^{P^{\prime}} \phi=\sum_{P_{1} \in \mathcal{P}_{P}^{P^{\prime}}} \varepsilon_{P_{1}}^{P_{1}^{\prime}} \Pi^{P_{1}} \phi
$$

for parabolic $F$-subgroups $P \subset P^{\prime}$. The ideas go back to Gelfand and PyatetskiShapiro [8]. 
For our present purposes, a Siegel domain with respect to a parabolic $F$ subgroup $P$ of $G$ is a set of the form

$$
\mathcal{S}=\left\{x \in \omega A(\mathbb{A}) K \mid H_{P}(x) \in \mathfrak{a}_{P}^{+}+T_{P}\right\}
$$

where $\omega$ is a compact subset of $P(\mathbb{A})^{1}, A$ is a maximal $F$-split torus in $P, T_{P} \in \mathfrak{a}_{P}$ and $K=K_{\infty} K_{\mathrm{f}}$ is the maximal compact subgroup of $G(\mathbb{A})$ fixed in section 1 . The right action of $G\left(F_{\infty}\right)$ on $G(\mathbb{A})$ associates to each element $X$ of the corresponding universal enveloping algebra $\mathfrak{G}$ a differential operator, which will be denoted by $X_{R}$.

Lemma 9 Let $P \subset P^{\prime}$ in $\mathcal{P}_{0}$ such that $P^{\prime} \neq G$ is maximal and denote by $\alpha \in \Delta_{P}$ the unique simple root not vanishing on $\mathfrak{a}_{P^{\prime}}$. Let $K_{0}$ be an open subgroup of $G\left(\mathbb{A}_{\mathrm{f}}\right)$, $\mathcal{S}$ a Siegel domain for $P$ and $r \in \mathbb{N}$. Then there exists a finite set $\mathfrak{F} \subset \mathfrak{G}$ such that, for all $\phi \in C^{\infty}\left(N^{\prime}(F) \backslash G(\mathbb{A}) / K_{0}\right)$ and $x \in \mathcal{S}$,

$$
\left|\phi(x)-\Pi^{P^{\prime}} \phi(x)\right| \leq e^{-r \alpha\left(H_{P}(x)\right)} \sup _{n^{\prime} \in N^{\prime}(\mathbb{A})} \max _{X \in \mathfrak{F}}\left|X_{R} \phi\left(n^{\prime} x\right)\right| .
$$

Of course, it would suffice to let the variable $n^{\prime}$ run through a fundamental domain for $N^{\prime}(F)$. It is customary, but unnecessary to rewrite the bound with the supremum over a set of right translations.

Since $\mathcal{S}$ is contained in a Siegel domain for $P_{0}$ and there exists $C_{1}>0$ such that $\alpha\left(H_{P}(x)\right) \leq \alpha_{0}\left(H_{0}(x)\right)+C_{1}$ for $x \in \mathcal{S}$, where $\alpha_{0}$ is the analogue of $\alpha$ for $P_{0}$, it is enough to prove the lemma for $P$ minimal. Variations on this result can be found in [9], ch. III $\S 8,[17]$, p. 45, [12], Lemma 2, [19], I.2.10 etc., so we will not repeat the proof. If $F$ is a function field (and $\mathfrak{G}=\mathbb{C}$ ), one can even show that there exists $C_{2}>0$ such that the left-hand side vanishes for $\alpha\left(H_{P}(x)\right)>C_{2}$ (cf. [19], I.2.7).

Lemma 10 Let $P \subset P^{\prime} \subset P^{\prime \prime}$ in $\mathcal{P}_{0}, K_{0}$ an open subgroup of $G\left(\mathbb{A}_{\mathrm{f}}\right), T_{P} \in \mathfrak{a}_{P}, \omega$ a compact subset of $P(\mathbb{A})^{1}$ and $\lambda$ a linear combination of the elements of $\Delta_{P}^{P^{\prime \prime}}-\Delta_{P}^{P^{\prime}}$ with non-negative coefficients. Then there exists a finite set $\mathfrak{F} \subset \mathfrak{G}$ such that, for all $\phi \in C^{\infty}\left(N^{\prime}(F) \backslash G(\mathbb{A}) / K_{0}\right)$ and all $x \in \omega A(\mathbb{A}) K$ satisfying

$$
\left(H_{P}(x)-T_{P}\right)^{P^{\prime \prime}} \in\left(\mathfrak{a}_{P}^{P^{\prime \prime}}\right)^{+}
$$

in the notation introduced in section 2 we have

$$
\left|\Pi_{P^{\prime}}^{P^{\prime \prime}} \phi(x)\right| \leq e^{-\lambda\left(H_{P}(x)\right)} \sup _{n^{\prime} \in N^{\prime}(\mathbb{A})} \max _{X \in \mathfrak{F}}\left|X_{R} \phi\left(n^{\prime} x\right)\right| .
$$

Proof. Actually, we prove the stronger inequality obtained upon replacing $\phi$ by $\Pi^{P^{\prime \prime}} \phi$. Thus assuming that $\phi$ is left $N^{\prime \prime}(\mathbb{A})$-invariant, we need only consider $\phi\left(m^{\prime \prime} k\right)$ as a function of $m^{\prime \prime} \in M^{\prime \prime}(\mathbb{A})$ for each $k \in K$, where $M^{\prime \prime}$ is a Levi component of $P^{\prime \prime}$. The intersection of the groups $M^{\prime \prime}(\mathbb{A}) \cap k K_{0} k^{-1}$ over $k \in$ 
$K_{\mathrm{f}} / K_{\mathrm{f}} \cap K_{0}$ is open in $M^{\prime \prime}(\mathbb{A})$, and the $K_{\infty}$-orbit of an element of $\mathfrak{M}^{\prime \prime}$ is contained in the span of a finite subset of $\mathfrak{G}$. Thus, by parabolic descent, we may assume w.l.o.g. that $P^{\prime \prime}=G$.

Now suppose for a moment that $P^{\prime}$ is maximal. Then $\Delta_{P}-\Delta_{P}^{P^{\prime}}=\{\alpha\}$ and $\lambda=r \alpha, r \geq 0$. The larger $r$ is, the stronger the estimate will be, and for $r \in \mathbb{N}$ it follows directly from Lemma 9. If $P^{\prime}$ is arbitrary, then

$$
\Pi_{P^{\prime}}^{G}=\prod_{P_{1}} \Pi_{P_{1}}^{G}
$$

where $P_{1}$ runs through the maximal parabolic $F$-subgroups containing $P^{\prime}$, hence the result follows upon iteration.

Replacing $G(\mathbb{A})$ by $G(\mathbb{A})^{1}$ leads to minor changes in the preceding discussion. In particular, the Siegel domain $\mathcal{S}$ has to be replaced by $\mathcal{S}^{1}=\mathcal{S} \cap G(\mathbb{A})^{1}$. We leave the details to the reader.

\section{$7 \quad$ Basic identities}

As in section 4, we fix a minimal parabolic $F$-subgroup $P_{0}=M_{0} N_{0}$ and its $F$ split component $A_{0}$. The truncated kernel $k_{\mathfrak{o}}^{T}$ was defined as a sum over parabolic $F$-subgroups $P$ containing $\mathcal{P}_{0}$ and over $\delta \in P(F) \backslash G(F)$, where in each term the variable $H_{P}(\delta x)$ was restricted to a shift of the cone ${ }^{+} \mathfrak{a}_{P}$. In order to show, with the aid of the results of the preceding section, that this kernel is rapidly decreasing in certain directions, we have to decompose that cone into the subsets

$$
\mathfrak{s}_{P}^{P^{\prime}}=\left\{H \in{ }^{+} \mathfrak{a}_{P} \mid \alpha(H)>0 \forall \alpha \in \Delta_{P}^{P^{\prime}} \text { and } \alpha(H) \leq 0 \forall \alpha \in \Delta_{P}-\Delta_{P}^{P^{\prime}}\right\}
$$

parametrised by the parabolic $F$-subgroups $P^{\prime}$ containing $P$ and, for each pair $P \subset P^{\prime}$, rewrite the kernel function in terms of alternating sums of constant terms of a fixed function. The argument of [1] is not sufficient here as it requires the test function to be compactly supported.

Let us recall some standard notation. We denote the characteristic function of the chamber $\mathfrak{a}_{P}^{+}$by $\tau_{P}$ and, as before, the characteristic function of the dual cone ${ }^{+} \mathfrak{a}_{P}$ by $\hat{\tau}_{P}$. More generally, if $P^{\prime} \supset P$ with a Levi component $M^{\prime}$ defined over $F$, we set $\tau_{P}^{P^{\prime}}=\tau_{P \cap M^{\prime}}$, which is the characteristic function of $\left(\mathfrak{a}_{P}^{P^{\prime}}\right)^{+}+\mathfrak{a}_{P^{\prime}}$, and $\hat{\tau}_{P}^{P^{\prime}}=\hat{\tau}_{P \cap M^{\prime}}$. We also extend these $\mathfrak{a}_{P^{\prime}}$-invariant functions on $\mathfrak{a}_{P}$ to $\mathfrak{a}_{0}^{P}$ invariant functions on $\mathfrak{a}_{0}=\mathfrak{a}_{0}^{P} \oplus \mathfrak{a}_{P}$. Finally, we denote by $\mathcal{P}_{P}^{P^{\prime}}$ the set of all parabolic $F$-subgroups $P_{1}$ with $P \subset P_{1} \subset P^{\prime}$, usually omitting the superscript if it equals $G$.

The results from the preceding section apply to restrictions of functions to a Siegel domain. This requires restricting certain components of the argument to compact sets, which will be granted by the appearance of a suitable cut-off 
function. Given $P^{\prime} \in \mathcal{P}_{0}:=\mathcal{P}_{P_{0}}$ and $T \in \mathfrak{a}_{0}$, we define

$$
F^{P^{\prime}}(x, T)=\sum_{P \in \mathcal{P}_{0}^{P^{\prime}}} \varepsilon_{P}^{P^{\prime}} \sum_{\delta \in P(F) \backslash P^{\prime}(F)} \hat{\tau}_{P}^{P^{\prime}}\left(H_{P}(\delta x)-T_{P}\right) .
$$

This $P^{\prime}(F) N^{\prime}(\mathbb{A}) A^{\prime}(\mathbb{A})$-invariant function on $G(\mathbb{A})$ is the result of applying the partial truncation operator $\Lambda^{T, P^{\prime}}$ (see [2], p. 97) to the constant function 1 . The inner sum, which can also be taken over $P(F) \cap M^{\prime}(F) \backslash M^{\prime}(F)$, is locally finite by Lemma 5.1 of [1]. For sufficiently regular $T$, one can alternatively define $F^{P^{\prime}}(x, T)$ as the characteristic function of a certain set. The next lemma shows that the two definitions are equivalent.

Lemma 11 (i) For any $T \in \mathfrak{a}_{0}$, we have the identity

$$
\sum_{P \in \mathcal{P}_{0}^{P^{\prime}}} \sum_{\delta \in P(F) \backslash P^{\prime}(F)} F^{P}(\delta x, T) \tau_{P}^{P^{\prime}}\left(H_{0}(\delta x)-T\right)=1 .
$$

(ii) For sufficiently regular $T \in \mathfrak{a}_{0}^{+}$, our function $F^{P}(x, T)$ coincides with that defined in [1], p. 941.

(iii) For $T, X \in \mathfrak{a}_{0}$, we have

$$
F^{P^{\prime}}(x, T+X)=\sum_{P \in \mathcal{P}_{0}^{P^{\prime}}} \sum_{\delta \in P(F) \backslash P^{\prime}(F)} F^{P}(\delta x, T) \Gamma_{P}^{P^{\prime}}\left(H_{0}(\delta x)-T, X\right),
$$

where we use the function

$$
\Gamma_{P}^{P^{\prime}}(H, X):=\sum_{Q \in \mathcal{P}_{P}^{P^{\prime}}} \varepsilon_{Q}^{P^{\prime}} \tau_{P}^{Q}(H) \hat{\tau}_{Q}^{P^{\prime}}(H-X)
$$

introduced in [3], p. 13.

(iv) As a function of $x, F^{P}(x, T)$ is both bounded and compactly supported modulo $P(F) N(\mathbb{A}) A(\mathbb{A})$ uniformly for $T$ varying in a compact set. Here $N$ is the unipotent radical and $A$ is some maximal $F$-split torus of $P$.

Proof. (i) On the left-hand side, we insert the definition of $F^{P}(x, T)$, in which we change the summation variable to $P_{1}$, say, and apply Langlands' Combinatorial Lemma (see [16], Lemma 5; [1], Lemma 6.3)

$$
\sum_{P \in \mathcal{P}_{P_{1}}^{P^{\prime}}} \varepsilon_{P}^{P^{\prime}} \hat{\tau}_{P_{1}}^{P}(H) \tau_{P}^{P^{\prime}}(H)= \begin{cases}1 & \text { if } P_{1}=P^{\prime} \\ 0 & \text { otherwise. }\end{cases}
$$

(ii) If we single out the term with $P=P^{\prime}$, the identity in (i) may be read as a recursive definition of $F^{P}(x, T)$. Due to [1], Lemma 6.4, it is also satisfied by 
the functions $F^{P}(x, T)$ defined in that paper for sufficiently regular $T$. Thus, the equality follows by induction.

(iii) By definition and the Combinatorial Lemma, $F^{P^{\prime}}(x, T+X)$ equals

$\sum_{P_{1} \subset P \subset P_{2}} \varepsilon_{P}^{P_{2}} \varepsilon_{P_{1}}^{P^{\prime}} \sum_{\delta \in P_{1}(F) \backslash P^{\prime}(F)} \hat{\tau}_{P_{1}}^{P_{1}}\left(H_{0}(\delta x)-T\right) \tau_{P}^{P_{2}}\left(H_{0}(\delta x)-T\right) \tau_{P_{2}}^{P^{\prime}}\left(H_{0}(\delta x)-T-X\right)$,

the exterior sum being taken over all triples $P_{1} \subset P \subset P_{2}$ in $\mathcal{P}_{0}^{P^{\prime}}$. The sum over $P_{2}$ can be taken innermost and simplified using the definition of $\Gamma_{P}^{P^{\prime}}$. Referring to the definition of $F^{P}(x, T)$ again, we obtain our assertion (which has also been proved in [18] for $X \in \mathfrak{a}_{0}^{+}$and sufficiently regular $T$ ).

(iv) For fixed sufficiently regular $T$, the support property follow from the definition given in [1], p. 941. Thus, for every $P$ there is a compact set $\omega_{1} \subset$ $G(\mathbb{A})$ such that the support of $F^{P}(x, T)$ is contained in $P(F) N(\mathbb{A}) A(\mathbb{A}) \omega_{1}$. By Lemma 2.1 of [3], the function $\Gamma_{P}^{P^{\prime}}(H, X)$ is compactly supported in $H \in \mathfrak{a}_{P}^{P^{\prime}}$ uniformly for $X$ varying in a compact set $\Omega$, say. Hence there is a compact set $\omega_{2} \subset A(\mathbb{A})$ such that the corresponding term on the right-hand side of (iii) is supported on $P^{\prime}(F) N(\mathbb{A}) A^{\prime}(\mathbb{A}) \omega_{2} \omega_{1}$ for $X \in \Omega$. And finally, there is a compact set $\omega_{3} \subset M^{\prime}(\mathbb{A}) \cap N(\mathbb{A})$ such that $N(\mathbb{A})=N(F) N^{\prime}(\mathbb{A}) \omega_{3}$. This shows that for sufficiently regular $T$ and compact $\Omega \subset \mathfrak{a}_{0}$ there exists a compact set $\omega \subset G(\mathbb{A})$ such that the support of each term on the right-hand side of (iii) is contained in $P^{\prime}(F) N^{\prime}(\mathbb{A}) A^{\prime}(\mathbb{A}) \omega$ for all $X \in \Omega$.

In order to prove the boundedness uniformly for $T \in \Omega$, it suffices to bound the number of terms in the sum defining $F^{P}(x, T)$ for a fixed $T$ such that $\Omega \subset{ }^{+} \mathfrak{a}_{0}+T$. As we noted earlier, Lemma 5.1 of [2] shows that this number is locally bounded, so it is bounded on the support of $F^{P}(x, T)$, because the latter is compact, as we have seen.

In order to state the identities figuring in the title of this section, it is best to introduce some new notation. For $\mathfrak{o} \in \mathcal{O}$ as in section 1 , for $x, y \in G(\mathbb{A})^{1}$ and for $w$ in the Weyl group $W_{0}$ of $A_{0}$ in $G$, we set

$$
L_{\mathfrak{o}}^{w}(x, y)=\sum_{\gamma \in G_{w}(F) \cap \mathfrak{o}} f\left(x^{-1} \gamma y\right) .
$$

If a parabolic $F$-subgroup $P^{\prime}$ contains both $P_{0}$ and ${ }^{w} P_{0}$, then $P^{\prime}={ }^{w} P^{\prime}$ and $G_{w}=M_{w}^{\prime} N^{\prime}$ (cf. the proof of Theorem 4). Since $P^{\prime}(F) \cap \mathfrak{o}=\left(M^{\prime}(F) \cap \mathfrak{o}\right) N^{\prime}(F)$, it follows that $G_{w}(F) \cap \mathfrak{o}$ is biinvariant under translations by $N^{\prime}(F)$. Hence the function $L_{\mathfrak{o}}^{w}$ is $N^{\prime}(F)$-invariant in each argument, and since $G_{w}$ normalises $N^{\prime}$, we have

$$
\left(\Pi^{P^{\prime}} \otimes \mathrm{Id}\right) L_{\mathfrak{o}}^{w}=\left(\mathrm{Id} \otimes \Pi^{P^{\prime}}\right) L_{\mathfrak{o}}^{w},
$$

where the tensor product with Id indicates to which argument of $L_{\mathfrak{o}}^{w}$ the constant term operator $\Pi^{P^{\prime}}$ is applied. 
Given $P \subset P^{\prime \prime}$ and $Q \subset Q^{\prime \prime}$ in $\mathcal{P}_{0}$, we further define

$$
K_{P, Q, \mathfrak{o}}^{P^{\prime \prime}, Q^{\prime \prime}}(x, y)=\sum_{w \in W_{0}}\left(\Pi_{P \vee^{w} Q^{\prime \prime} Q^{\prime \prime}}^{P^{\prime \prime}} \otimes \mathrm{Id}\right) L_{\mathfrak{o}}^{w}(x, y)
$$

where we use the operations $\vee$ and $\wedge$ introduced in section 2 . If the parabolic subgroup $P^{\prime \prime} \wedge{ }^{w} Q^{\prime \prime}$ exists and contains $P \vee{ }^{w} Q$, the operator $\Pi_{P \vee{ }^{\prime}}^{P^{\prime \prime}} Q^{\prime \prime}$ has been defined in section 6 . (In this case, by the way, ${ }^{w} Q^{\prime \prime}=Q^{\prime \prime}$.) In any other case, we set this operator equal to zero. Equivalently, we could have restricted summation to the appropriate subset of $W_{0}$. We also use the notation

$$
K_{P, \mathfrak{o}}^{P^{\prime \prime}}(x)=K_{P, P, \mathfrak{o}}^{P^{\prime \prime}, P^{\prime \prime}}(x, x), \quad K_{P, Q}^{P^{\prime \prime}, Q^{\prime \prime}}=\sum_{\mathfrak{o} \in \mathcal{O}} K_{P, Q, \mathfrak{o}}^{P^{\prime \prime}, Q^{\prime \prime}} .
$$

The first of these functions is left-invariant under $P(F)$, the second one-under $P(F) \times Q(F)$, whereas its $\mathfrak{o}$-components will not be so in general.

Theorem 5 Let $f \in L^{1}\left(G(\mathbb{A})^{1}\right), \mathfrak{o} \in \mathcal{O}, T \in \mathfrak{a}_{0}$ and $x, y \in G(\mathbb{A})$. Then

$$
k_{\mathfrak{o}}^{T}(x)=\sum_{P \subset P^{\prime \prime}} \sum_{\delta \in P(F) \backslash G(F)} F^{P}(\delta x, T) \sigma_{P}^{P^{\prime \prime}}\left(H_{P}(\delta x)-T_{P}\right) K_{P, \mathfrak{o}}^{P^{\prime \prime}}(\delta x),
$$

provided the sum over $\delta$ is absolutely convergent, and

$$
\begin{aligned}
k^{T}(x, y)=\sum_{\substack{P \subset P^{\prime \prime} \\
Q \subset Q^{\prime \prime}}} \sum_{\substack{\delta \in P(F) \backslash G(F) \\
\eta \in Q(F) \backslash G(F)}} F^{P}(\delta x, T) \sigma_{P}^{P^{\prime \prime}}\left(H_{P}(\delta x)-T_{P}\right) \\
\quad \times F^{Q}(\eta y, T) \sigma_{Q}^{Q^{\prime \prime}}\left(H_{Q}(\eta y)-T_{Q}\right) K_{P, Q}^{P^{\prime \prime}, Q^{\prime \prime}}(\delta x, \eta y),
\end{aligned}
$$

provided the sum over $\delta$ and $\eta$ is absolutely convergent. Here, the exterior sums run over the indicated configurations of elements of $\mathcal{P}_{0}$, and $\sigma_{P}^{P^{\prime \prime}}$ denotes, as usual, the characteristic function of the set $\mathfrak{s}_{P}^{P^{\prime \prime}}$.

Proof. Ignoring questions of convergence for the moment, we insert the formula from Lemma 11(i) in the definition of $k_{\mathfrak{o}}^{T}(x)$, which yields

$$
\sum_{P \subset P^{\prime}} \varepsilon_{P^{\prime}}^{G} \sum_{\delta \in P(F) \backslash G(F)} F^{P}(\delta x, T) \tau_{P}^{P^{\prime}}\left(H_{0}(\delta x)-T\right) \hat{\tau}_{P^{\prime}}\left(H_{0}(\delta x)-T\right) K_{\mathfrak{o}}^{P^{\prime}}(\delta x, \delta x) .
$$

By [1], Lemma 6.1,

$$
\tau_{P}^{P^{\prime}}(H) \hat{\tau}_{P^{\prime}}(H)=\sum_{P^{\prime \prime} \in \mathcal{P}_{P^{\prime}}} \sigma_{P}^{P^{\prime \prime}}(H)
$$

where $\sigma_{P}^{P^{\prime \prime}}$ has been extended to $\mathfrak{a}_{0}$ in the customary way, and we get

$$
k_{\mathfrak{o}}^{T}(x)=\sum_{P \subset P^{\prime} \subset P^{\prime \prime}} \varepsilon_{P^{\prime}}^{G} \sum_{\delta \in P(F) \backslash G(F)} F^{P}(\delta x, T) \sigma_{P}^{P^{\prime \prime}}\left(H_{0}(\delta x)-T\right) K_{\mathfrak{o}}^{P^{\prime}}(\delta x, \delta x) .
$$


So far we have followed [1], p. 942/943. Now we claim that $k_{\mathfrak{o}}^{T}(x)$ equals

$$
\sum_{P \subset P_{1} \subset P^{\prime} \subset P_{2} \subset P^{\prime \prime}} \varepsilon_{P_{1}}^{P^{\prime}} \varepsilon_{P_{2}}^{G} \sum_{\delta \in P(F) \backslash G(F)} F^{P}(\delta x, T) \sigma_{P}^{P^{\prime \prime}}\left(H_{0}(\delta x)-T\right)\left(\Pi^{P_{2}} \otimes \operatorname{Id}\right) L_{\mathfrak{o}}^{P_{1}}(\delta x, \delta x),
$$

where

$$
L_{\mathfrak{o}}^{P_{1}}(x, y)=\sum_{\gamma \in P_{1}(F) \cap \mathfrak{o}} f\left(x^{-1} \gamma y\right) .
$$

This follows easily upon application of the identity

$$
\sum_{P^{\prime} \in \mathcal{P}_{P_{1}}^{P_{2}}} \varepsilon_{P_{1}}^{P^{\prime}}= \begin{cases}1 & \text { if } P_{1}=P_{2} \\ 0 & \text { otherwise }\end{cases}
$$

(see [1], Prop. 1.1), if one notes that $K_{\mathfrak{o}}^{P^{\prime}}=\left(\Pi^{P^{\prime}} \otimes \mathrm{Id}\right) L_{\mathfrak{o}}^{P^{\prime}}$.

Multiplying the Bruhat decomposition of $M_{1}$ through with $N_{1}$, we get

$$
P_{1}(F)=\bigcup_{w \in W_{0}^{M_{1}}} P_{0}(F) \tilde{w} P_{0}(F) \quad \text { (disjoint union), }
$$

which implies that

$$
L_{\mathfrak{o}}^{P_{1}}=\sum_{w \in W_{0}^{M_{1}}} L_{\mathfrak{o}}^{w}=\sum_{\substack{w \in W_{0} \\ P_{1}={ }^{w} P_{1}}} L_{\mathfrak{o}}^{w}
$$

and therefore

$$
\sum_{P_{1} \in \mathcal{P}_{P}^{P^{\prime}}} \varepsilon_{P_{1}}^{P} L_{\mathfrak{o}}^{P_{1}}=\sum_{w \in W_{0}} L_{\mathfrak{o}}^{w} \sum_{\substack{P_{1} \in \mathcal{P}_{P}^{\prime} \\ P_{1}=P_{1}^{\prime}}} \varepsilon_{P_{1}}^{P^{\prime}}
$$

The interior sum depends only on the image of $w$ in $W_{A}$, where $A$ is the maximal $F$-split torus in $P$ containing $A_{0}$. Due to Lemma 2(iv), this sum runs over $\mathcal{P}_{P_{1} \vee{ }^{w} P_{1}}^{P^{\prime}}$. By (9), it vanishes unless $P^{\prime}=P_{1} \vee{ }^{w} P_{1}$, and we formally obtain the first identity claimed in the theorem.

If the sum over $\delta$ on the right-hand side is absolutely convergent, we may rightfully perform all transformations in the opposite direction to arrive at the definition of $k_{\mathfrak{o}}^{T}(x)$.

The proof of the second formula is similar. Using Lemma 11(i) and (8), we get

$$
\begin{aligned}
k^{T}(x, y)= & \sum_{\substack{P \subset P^{\prime} \subset P^{\prime \prime} \\
Q \subset P^{\prime} \subset Q^{\prime \prime}}} \sum_{\substack{\delta \in P(F) \backslash G(F) \\
\eta \in Q(F) \backslash G(F)}} F^{P}(\delta x, T) \sigma_{P}^{P^{\prime \prime}}\left(H_{0}(\delta x)-T\right) \\
& \times F^{Q}(\eta y, T) \sigma_{Q}^{Q^{\prime \prime}}\left(H_{0}(\eta y)-T\right) K^{P^{\prime}}(\delta x, \eta y) .
\end{aligned}
$$


Again we apply (9) to transform this into

$$
\begin{aligned}
\sum_{\substack{P \subset P_{1} \subset P^{\prime} \subset P_{2} \subset P^{\prime \prime} \\
Q \subset P_{1} \subset P^{\prime} \subset P_{2} \subset Q^{\prime \prime}}} \varepsilon_{P_{1}}^{P^{\prime}} \varepsilon_{P_{2}}^{G} \sum_{\substack{\delta \in P(F) \backslash G(F) \\
\eta \in Q(F) \backslash G(F)}} F^{P}(\delta x, T) \sigma_{P}^{P^{\prime \prime}}\left(H_{0}(\delta x)-T\right) \\
\quad \times F^{Q}(\eta y, T) \sigma_{Q}^{Q^{\prime \prime}}\left(H_{0}(\eta y)-T\right)\left(\Pi^{P_{2}} \otimes \operatorname{Id}\right) L^{P_{1}}(\delta x, \eta y),
\end{aligned}
$$

where $L^{P_{1}}=\sum_{\mathfrak{o}} L_{\mathfrak{o}}^{P_{1}}$. As before, we decompose $L^{P_{1}}$ into a sum over $w \in W_{0}$ such that $P_{1}={ }^{w} P_{1}$ and observe that

$$
\sum_{\substack{P_{1} \in \mathcal{P}_{P}^{P^{\prime}} \cap \mathcal{P}_{Q}^{P^{\prime}} \\ P_{1}={ }^{\prime} P_{1}}} \varepsilon_{P_{1}}^{P^{\prime}}=\sum_{\substack{P_{1} \in \mathcal{P}_{P \vee}^{P^{\prime}} w_{Q} \\ P_{P_{1}}}} \varepsilon^{P^{\prime}}
$$

because if $P_{1} \supset P$ and $P_{1} \supset{ }^{w} Q$, then $P_{1} \supset P_{0}$ and $P_{1} \supset{ }^{w} P_{0}$, hence $P_{1}={ }^{w} P_{1}$ by Lemma $2(\mathrm{iv})$. As in the case of the first formula, another application of (9) completes the proof.

\section{Decay on $P(F) \backslash G(\mathbb{A})^{1}$}

Here we estimate a generic term on the right-hand side of the basic identities given in Theorem 5 .

Theorem 6 There exist constants $c>0$ and $d$ with the following property. Given a set $S$ of places of $F$ containing all infinite ones, an open compact subgroup $K_{0} \subset G\left(F_{S}\right)$, a truncation parameter $T \in \mathfrak{a}_{0}, 0<p<1$ and large $r$, we can find a finite set $\mathfrak{F} \subset \mathfrak{G}^{1} \times \mathfrak{G}^{1}$ such that, for all $f \in \mathcal{C}_{K_{0}}^{p}\left(G\left(F_{S}\right) K^{S}\right)$, subgroups $P \subset P^{\prime \prime}$ and $Q \subset Q^{\prime \prime}$ in $\mathcal{P}_{0}$ and $x, y \in G(\mathbb{A})^{1}$ we have that

$$
F^{P}(x, T) \sigma_{P}^{P^{\prime \prime}}\left(H_{0}(x)-T\right) \neq 0
$$

implies

$$
\sum_{\mathfrak{o} \in \mathcal{O}}\left|K_{P, \mathfrak{o}}^{P^{\prime \prime}}(x)\right| \leq\|f\|_{p, \mathfrak{F}, r} e^{-2\left(\frac{c}{p}-d\right)\left|H_{P}(x)\right|}
$$

and that

$$
F^{P}(x, T) \sigma_{P}^{P^{\prime \prime}}\left(H_{0}(x)-T\right) F^{Q}(y, T) \sigma_{Q}^{Q^{\prime \prime}}\left(H_{0}(y)-T\right) \neq 0
$$

implies

$$
\left|K_{P, Q}^{P^{\prime \prime}, Q^{\prime \prime}}(x, y)\right| \leq\|f\|_{p, \mathfrak{F}, r} e^{-\left(\frac{c}{p}-d\right)\left(\left|H_{P}(x)\right|+\left|H_{Q}(y)\right|\right)} .
$$

Remember that the left-hand side of either inequality depends implicitly on $f$. Both sides of the first estimate are left $P(F)$-invariant, and we have to consider only those $x$ for which $F^{P}(x, T) \neq 0$. Due to Lemma 11(iv), we may thus assume 
that $x$ belongs to $N(\mathbb{A}) A(\mathbb{A}) \omega_{1}$ for some compact subset $\omega_{1}$ of $G(\mathbb{A})$, or better to $N(\mathbb{A}) A(\mathbb{A}) \omega_{2} K=N(\mathbb{A}) \omega_{2} A(\mathbb{A}) K$ for some compact subset $\omega_{2}$ of $M(\mathbb{A})^{1}$, or even to $\omega A(\mathbb{A}) K$, where $\omega$ is a compact subset of $P(\mathbb{A})^{1}$. Similarly, both sides of the second estimate are left $P(F) \times Q(F)$-invariant, and we may assume in addition that $y \in \chi B(\mathbb{A}) K$, where $B$ is a maximal $F$-split torus in $Q$ and $\chi$ is a compact subset of $Q(\mathbb{A})^{1}$.

We are going to prove that, under these restrictions on $x$ and $y$, the second inequality remains valid if we replace the left-hand side by

$$
\sum_{\mathfrak{o} \in \mathcal{O}}\left|K_{P, Q, \mathfrak{o}}^{P^{\prime \prime}, Q^{\prime \prime}}(x, y)\right|
$$

(which is not $P(F) \times Q(F)$-invariant in general). After the foregoing discussion it is clear that the two estimates in the Theorem are then corollaries. Actually we are going to prove that the absolute value can even be taken separately of each term in the sum over $W_{0}$ which makes up $K_{P, Q, 0}^{P^{\prime \prime}, Q^{\prime \prime}}$. It suffices to consider the contribution from a fixed $w$ for which the parabolic subgroup $P_{2}:=P^{\prime \prime} \wedge Q^{\prime \prime}$ exists and contains $P_{1}:=P \vee{ }^{w} Q$. In this notation, our task is to bound

$$
\sum_{\mathfrak{o} \in \mathcal{O}}\left|\left(\Pi_{P_{1}}^{P_{2}} \otimes \mathrm{Id}\right) L_{\mathfrak{o}}^{w}(x, y)\right| .
$$

The condition $\sigma_{P}^{P^{\prime \prime}}\left(H_{0}(x)-T\right) \neq 0$ implies

$$
\left(H_{P}(x)-T_{P}\right)^{P^{\prime \prime}} \in\left(\mathfrak{a}_{P}^{P^{\prime \prime}}\right)^{+}
$$

and, since $P_{2} \subset P^{\prime \prime}$, the same inclusion with $P^{\prime \prime}$ replaced by $P_{2}$. Thus, for every linear combination $\lambda$ of the elements of $\Delta_{P}^{P_{2}}-\Delta_{P}^{P_{1}}$ with nonnegative coefficients, Lemma 10 supplies a finite set $\mathfrak{F}_{\lambda} \subset \mathfrak{G}^{1}$ such that

$$
\left|\left(\Pi_{P_{1}}^{P_{2}} \otimes \mathrm{Id}\right) L_{\mathfrak{o}}^{w}(x, y)\right| \leq e^{-\lambda\left(H_{P}(x)\right)} \sup _{n \in N_{1}(\mathbb{A})} \max _{X \in \mathfrak{F}_{\lambda}}\left|\left(X_{R} \otimes \mathrm{Id}\right) L_{\mathfrak{o}}^{w}(n x, y)\right| .
$$

By the triangle inequality, our condition on $f$ and the definition of $\kappa_{S, p, r}^{w}$,

$$
\sum_{\mathfrak{o} \in \mathcal{O}}\left|\left(X_{R} \otimes \mathrm{Id}\right) L_{\mathfrak{o}}^{w}(n x, y)\right| \leq \sum_{\gamma \in G_{w}(F)}\left|X_{L} f\left((n x)^{-1} \gamma y\right)\right| \leq\|f\|_{p,(X, 1), r} \kappa_{S, p, r}^{w}(n x, y) .
$$

Similarly, for every linear combination $\mu$ of the elements of $\Delta_{Q}^{P_{2}}-\Delta_{Q}^{P_{1}}$ with nonnegative coefficients, we can find a finite set $\mathfrak{F}_{\mu} \subset \mathfrak{G}^{1}$ such that the kernel $\left(\mathrm{Id} \otimes \Pi_{P_{1}}^{P_{2}}\right) L_{\mathfrak{o}}^{w}(x, y)$ has an analogous bound. However, both kernels coincide due to equation (7), and if we multiply the two inequalities, we get

$$
\begin{aligned}
\left(\sum_{\mathfrak{o} \in \mathcal{O}} \mid\left(\Pi_{P_{1}}^{P_{2}} \otimes \mathrm{Id}\right)\right. & \left.L_{\mathfrak{o}}^{w}(x, y) \mid\right)^{2} \leq e^{-\lambda\left(H_{P}(x)\right)-\mu\left(H_{Q}(y)\right)} \\
& \times\|f\|_{p, \mathfrak{F}_{\lambda} \times\{1\}, r}\|f\|_{p,\{1\} \times \mathfrak{F}_{\mu}, r} \sup _{n, u \in N_{1}(\mathbb{A})} \kappa_{S, p, r}^{w}(n x, y) \kappa_{S, p, r}^{w}(x, u y) .
\end{aligned}
$$


Here we take the square root and note that, by the Cauchy-Schwarz inequality,

$$
\|f\|_{p, \mathfrak{F}_{\lambda} \times\{1\}, r}\|f\|_{p,\{1\} \times \mathfrak{F}_{\mu}, r} \leq\|f\|_{p, \mathfrak{F}, r}^{2},
$$

where $\mathfrak{F}=\mathfrak{F}_{\lambda} \times\{1\} \cup\{1\} \times \mathfrak{F}_{\mu}$.

Let $P^{\prime}=P_{0} \vee^{w} P_{0}$. Clearly $P^{\prime} \subset P_{1}$ and therefore $P^{\prime} \subset P^{\prime \prime}$ and $P^{\prime} \subset Q^{\prime \prime}$. (See the diagram for the various inclusions of parabolics.)

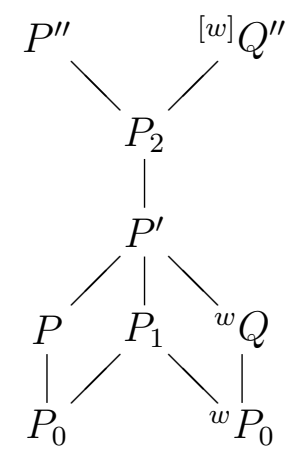

Due to the restriction imposed on $x$ and $y$, the projections $H_{0}(x)^{P}$ and $H_{0}(y)^{Q}$ (in the notation of section 2) remain in compact sets, while

$$
\left(H_{P}(x)-T_{P}\right)^{P^{\prime \prime}} \in \overline{\left(\mathfrak{a}_{P}^{P^{\prime \prime}}\right)^{+}}, \quad\left(H_{Q}(y)-T_{Q}\right)^{Q^{\prime \prime}} \in \overline{\left(\mathfrak{a}_{Q}^{Q^{\prime \prime}}\right)^{+}} .
$$

Projecting further, we see that the latter inclusions remain true with $P^{\prime \prime}$ and $Q^{\prime \prime}$ replaced by $P^{\prime}$. Therefore there exists $T_{1} \in \mathfrak{a}_{0}$ such that

$$
\left(H_{0}(x)-T_{1}\right)^{P^{\prime}},\left(H_{0}(y)-T_{1}\right)^{P^{\prime}} \in \overline{\left(\mathfrak{a}_{0}^{P^{\prime}}\right)^{+}} .
$$

The corollary of Theorem 4 now provides constants $c_{1}$ and $C_{1}$ such that

$$
\kappa_{S, p, r}^{w}(x, y) \leq C_{1} e^{-c_{1}\left(\frac{2}{p}-1\right)\left|H_{P}(x)-w H_{Q}(y)\right|+\left|H_{P}(x)\right|+\left|H_{Q}(y)\right|}
$$

for all $x, y$ as above, where $c_{1}$ is independent of $S, T, p$ and $r$. We combine this inequality with the preceding one and remark that its right-hand side is unchanged if we replace $x$ by $n x$ and $y$ by $u y$ with $n, u \in N_{1}(\mathbb{A})$. At the cost of enlarging $C_{1}$ (which will be absorbed into $\mathfrak{F}$ ) we may replace $H_{P}(x)$ by $H_{P}(x)-$ $T_{P} \in \mathfrak{s}_{P}^{P^{\prime \prime}}$ and $H_{Q}(y)$ by $H_{Q}(y)-T_{Q} \in \mathfrak{s}_{Q}^{Q^{\prime \prime}}$. Now it is clear that the following lemma serves to complete the proof of the theorem with $c=\max \left(2 c_{1} c_{2}, q\right)$ and $d=c+1$.

Lemma 12 In the above notations, we can choose $\lambda, \mu$ and a positive constant $c_{2}$ such that

$$
|U-w V|+\lambda(U)+\mu(V) \geq c_{2}(|U|+|V|)
$$

for all $U \in \overline{\mathfrak{s}_{P}^{P^{\prime \prime}}}$ and $V \in \overline{\mathfrak{s}_{Q}^{Q^{\prime \prime}}}$. 
Proof. Both sides of the inequality are seminorms on $\mathfrak{a}_{P} \times \mathfrak{a}_{Q}$ invariant under translations by $\mathfrak{a}_{G} \times \mathfrak{a}_{G}$. If we pass to the quotient by that subspace, the righthand side becomes a norm, and the projection of $\overline{\mathfrak{s}_{P}^{P \prime}} \times \overline{\mathfrak{s}_{Q}^{Q^{\prime \prime}}}$ intersects the corresponding unit sphere in a compact set. Hence it suffices to show that the vanishing of the left-hand side for the vectors $U$ and $V$ in question implies $(U, V) \in \mathfrak{a}_{G} \times \mathfrak{a}_{G}$.

The roots in $\Delta_{P}^{P_{2}}$, which is a subset of $\Delta_{P}^{P^{\prime \prime}}$, are all non-negative on $\overline{\mathfrak{s}_{P}^{P^{\prime \prime}}}$, hence so is $\lambda$. Similarly, $\mu$ is non-negative on $\overline{\mathfrak{s}_{Q}^{Q^{\prime \prime}}}$. Consequently, if the left-hand side of our inequality vanishes, so do all three terms.

The vanishing of the first term means that $U-w V \in \mathfrak{a}_{G}$. By the preceding step, the projection of $\overline{\mathfrak{s}_{P}^{P^{\prime \prime}}}$ onto $\mathfrak{a}_{0}^{P_{2}}$ is contained in $\overline{\left(\mathfrak{a}_{P}^{P_{2}}\right)^{+}}$, and keeping the definition of $P_{1}$ in mind, we conclude that

$$
H^{P_{2}} \in \overline{\left(\mathfrak{a}_{P}^{P_{2}}\right)^{+}} \cap \overline{w\left(\mathfrak{a}_{Q}^{P_{2}}\right)^{+}}=\overline{\left(\mathfrak{a}_{P_{1}}^{P_{2}}\right)^{+}} .
$$

On the other hand, the restriction of $\Delta_{P}^{P_{2}}-\Delta_{P}^{P_{1}}$ to $\mathfrak{a}_{P_{1}}^{P_{2}}$ equals $\Delta_{P_{1}}^{P_{2}}$, which is a basis of that space, so we can choose $\lambda$ such that it is positive on $\overline{\left(\mathfrak{a}_{P_{1}}^{P_{2}}\right)^{+}}-\{0\}$. Since

$$
\lambda\left(H^{P_{2}}\right)=\lambda(H)=0
$$

it follows that $H \in \mathfrak{a}_{P_{2}}$.

The restriction of $\Delta_{P}^{P^{\prime \prime}}$ to $\mathfrak{a}_{P_{2}}$ being $\Delta_{P_{2}}^{P^{\prime \prime}} \cup\{0\}$, we have $\overline{\mathfrak{s}_{P}^{P^{\prime \prime}}} \cap \mathfrak{a}_{P_{2}}=\overline{\mathfrak{s}_{P_{2}}^{P^{\prime \prime}}}$, and the condition $U-w V \in \mathfrak{a}_{G}$ together with ${ }^{w} Q^{\prime \prime}=Q^{\prime \prime}$ entails

$$
U \in \overline{\mathfrak{s}_{P_{2}}^{P^{\prime \prime}}} \cap \overline{\mathfrak{s}_{P_{2}}^{Q^{\prime \prime}}} .
$$

The oppositional inequalitites defining these sets imply that $\alpha(U)=0$ for $\alpha \in$ $\Delta_{P_{2}}^{P^{\prime \prime}}-\Delta_{P_{2}}^{Q^{\prime \prime}}$ or $\alpha \in \Delta_{P_{2}}^{Q^{\prime \prime}}-\Delta_{P_{2}}^{P^{\prime \prime}}$. Note that the definition $P_{2}=P^{\prime \prime} \wedge Q^{\prime \prime}$ can be rephrased as $\Delta_{P_{2}}^{P^{\prime \prime}} \cap \Delta_{P_{2}}^{Q^{\prime \prime}}=\emptyset$, while the parabolic $P_{3}:=P^{\prime \prime} \vee Q^{\prime \prime}$ is characterised by $\Delta_{P_{2}}^{P_{3}}=\Delta_{P_{2}}^{P^{\prime \prime}} \cup \Delta_{P_{2}}^{Q^{\prime \prime}}$. Thus, we have $\alpha(U)=0$ for $\alpha \in \Delta_{P_{2}}^{P_{3}}$, in other words, $U \in \mathfrak{a}_{3}$.

On the one hand, we know that $U \in \overline{\mathfrak{s}_{P_{2}}} \subset \overline{{ }^{+} \mathfrak{a}_{P_{2}}}$, hence $U \in \overline{{ }^{+} \mathfrak{a}_{P_{3}}}$. However, the restriction of $\Delta_{P_{2}}^{P^{\prime \prime}}-\Delta_{P_{2}}^{P_{3}}$ onto $\mathfrak{a}_{P_{3}}$ is $\Delta_{P_{3}}^{P^{\prime \prime}}$, so the inequalities

$$
\alpha(U) \leq 0 \quad \text { for } \alpha \in \Delta_{P_{3}}^{P^{\prime \prime}} \cup \Delta_{P_{3}}^{Q^{\prime \prime}}=\Delta_{P_{3}}
$$

are among those defining $\overline{\mathfrak{s}_{P_{2}}}$ and $\overline{\mathfrak{s}_{P_{2}}^{Q^{\prime \prime}}}$. Thus, on the other hand, $-U \in \overline{\mathfrak{a}_{P_{3}}^{+}} \subset$ $\overline{{ }^{+} \mathfrak{a}_{P_{3}}}$. Since the projection of ${ }^{+} \mathfrak{a}_{P_{3}}$ onto $\mathfrak{a}_{P_{3}}^{G}$ is a proper cone, we conclude that $U \in \mathfrak{a}_{G}$.

Swapping $P, P^{\prime \prime}, U, w$ with $Q, Q^{\prime \prime}, V, w^{-1}$, resp., we see that $V \in \mathfrak{a}_{G}$ as well. 


\section{Proof of the main results}

We know from Theorem 5 that

$$
\left|k_{\mathfrak{o}}^{T}(x)\right| \leq \sum_{P \subset P^{\prime \prime}} \sum_{\delta \in P(F) \backslash G(F)} \sigma_{P}^{P^{\prime \prime}}\left(H_{P}(\delta x)-T_{P}\right)\left|F^{P}(\delta x, T) K_{P, \mathfrak{o}}^{P^{\prime \prime}}(\delta x)\right|
$$

as soon as the right-hand side converges, keeping in mind that $\sigma_{P}^{P^{\prime \prime}}$ takes on values 0 and 1 only. In order to prove Theorem 1 , we integrate over $G(F) \backslash G(\mathbb{A})^{1}$ and combine the integral on the right-hand side with the sum over $P(F) \backslash G(F)$ (after swapping it with the finite sum over $P$ and $P^{\prime \prime}$ ) to get

$$
\begin{aligned}
& \int_{G(F) \backslash G(\mathbb{A})^{1}}\left|k_{\mathfrak{o}}^{T}(x)\right| d x \\
& \leq \sum_{P \subset P^{\prime \prime}} \int_{P(F) \backslash G(\mathbb{A})^{1}} \sigma_{P}^{P^{\prime \prime}}\left(H_{P}(x)-T_{P}\right)\left|F^{P}(x, T) K_{P, \mathfrak{o}}^{P^{\prime \prime}}(x)\right| d x
\end{aligned}
$$

provided the last integral converges. We have to bound the sum of this expression over all $\mathfrak{o} \in \mathcal{O}$. Due to Theorem 6 ,

$$
\begin{aligned}
& \sum_{\mathfrak{o} \in \mathcal{O}} \int_{P(F) \backslash G(\mathbb{A})^{1}} \sigma_{P}^{P^{\prime \prime}}\left(H_{P}(x)\right.\left.-T_{P}\right)\left|F^{P}(x, T) K_{P, \mathfrak{o}}^{P^{\prime \prime}}(x)\right| d x \\
& \leq C_{1}\|f\|_{p, \mathfrak{F}, r} \int_{P(F) \backslash G(\mathbb{A})^{1}}\left|F^{P}(x, T)\right| e^{-2\left(\frac{c}{p}-d\right)\left|H_{P}(x)\right|} d x,
\end{aligned}
$$

where $C_{1}$ depends on the bound from Lemma 11(iv). The usual integration formula shows that for suitably normalised measures the last integral equals

$$
\int_{M(F) \backslash M(\mathbb{A})^{1}}\left|F^{M}(m, T)\right| d m \int_{\mathfrak{a}_{P}^{G}} e^{-2 \rho_{P}(H)-2\left(\frac{c}{p}-d\right)|H|} d H,
$$

which is finite for sufficiently small $p>0$ by Lemma 11(iv) and Lemma 1(i). This proves Theorem 1. (To improve the range of $p$, we could have replaced $\left|H_{P}(x)\right|$ by $\left|H_{P}(x)-T_{P}\right|$ at the cost of $C_{1}$ and integrated over ${ }^{+} \mathfrak{a}_{P}^{G}$ only.)

The proof of Theorem 2 starts with the same inequality (10), where we immediately substitute the bound from Theorem 6 . Summing over $\mathcal{O}$, we get

$$
\sum_{\mathfrak{o} \in \mathcal{O}}\left|k_{\mathfrak{o}}^{T}(x)\right| \leq C_{1}\|f\|_{p, \mathfrak{F}, r} \sum_{P \in \mathcal{P}_{0}} B_{P, 2\left(\frac{c}{p}-d\right)}^{T}(x),
$$

provided the series

$$
B_{P, t}^{T}(x):=\sum_{\delta \in P(F) \backslash G(F)}\left|F^{P}(\delta x, T)\right| \hat{\tau}_{P}\left(H_{P}(\delta x)-T_{P}\right) e^{-t\left|H_{P}(\delta x)\right|}
$$


converges for $t=2\left(\frac{c}{p}-d\right)$.

Before completing the proof, let us remark that Theorem 3 can be treated in exactly the same way. We know from Theorem 6 that

$$
\begin{aligned}
\left|k^{T}(x, y)\right| \leq \sum_{\substack{P \subset P^{\prime \prime} \\
Q \subset Q^{\prime \prime}}} \sum_{\delta \in P(F) \backslash G(F)} \mid F^{P}(\delta x, T) \sigma_{P}^{P^{\prime \prime}}\left(H_{P}(\delta x)-T_{P}\right) \\
\times F^{Q}(\eta y, T) \sigma_{Q}^{Q^{\prime \prime}}\left(H_{Q}(\eta y)-T_{Q}\right) K_{P, Q}^{P^{\prime \prime}, Q^{\prime \prime}}(\delta x, \eta y) \mid,
\end{aligned}
$$

if we assume the convergence of the right-hand side, which in turn is bounded, due to Theorem 6, by

$$
C_{2}\|f\|_{p, \mathfrak{\xi}, r} \sum_{P, Q \in \mathcal{P}_{0}} B_{P, \frac{c}{p}-d}^{T}(x) B_{Q, \frac{c}{p}-d}^{T}(y) .
$$

It is clear that the following result suffices to complete the proof of Theorems 2 and 3 .

Lemma 13 Given $P \in \mathcal{P}_{0}, T \in \mathfrak{a}_{0}$ and any real number $t_{0}$, there exist $a>0, b$ and $C$ such that, for all $t>t_{0}$ and $x \in \mathcal{S}_{0}$,

$$
\left|B_{P, t}^{T}(x)\right| \leq C e^{(b-a t) \rho_{0}\left(H_{0}(x)\right)} .
$$

The proof of this lemma is a variation on that of Lemma 4.1 in [2]. Let us fix a maximal $F$-split torus $A$ in $P$, a height function on $G(\mathbb{A})$ as in [19], section I.2.2, and a valuation $v_{0}$ of $F$ as in the proof of Lemma 7 .

By Lemma 11(iv) and the compactness of $P(F) \backslash P(\mathbb{A})^{1}$, there exists a compact subset $\omega \subset P(\mathbb{A})$ such that $F^{P}(x, T)=0$ unless $x \in P(F) \omega A\left(F_{v_{0}}\right) K$. We claim that there exist $C$ and $c>0$ such that

$$
\|y\| \leq C e^{c\left|H_{P}(y)\right|}
$$

for all $y \in \omega A\left(F_{v_{0}}\right) K$. In view of the property $\left\|y_{1} y_{2}\right\| \leq\left\|y_{1}\right\|\left\|y_{2}\right\|$ of the height function it suffices to check this for $y \in A\left(F_{v_{0}}\right)$, in which case it follows from property (iv) of the height function (which is stated in [19] for a slightly different group but holds for $A\left(F_{v_{0}}\right)$, too, while it would fail for $A(\mathbb{A})$ ).

Given $x \in G(\mathbb{A})$ with $F^{P}(x, T) \neq 0$ and a coset $P(F) \delta$ in $G(F)$, we may thus choose the representative $\delta$ in such a way that $\delta x \in \omega A\left(F_{v_{0}}\right) K$, which allows us to bound $\left|B_{P, t}^{T}(x)\right|$ for $t \geq 0$ by a similar expression in which $e^{-t\left|H_{P}(\delta x)\right|}$ has been replaced by $\|\delta x\|^{-t / c}$. If we define a modified Siegel domain with respect to $P_{0}$ as

$$
\mathcal{S}_{0}^{\prime}=\left\{x \in \omega_{0} A_{0}\left(F_{v_{0}}\right) K \mid H_{0}(x) \in \mathfrak{a}_{0}^{+}+T_{0}\right\},
$$

where $\omega_{0}$ is a compact subset of $P_{0}(\mathbb{A})$, then property (vii) of the height function (see [19], whose proof applies here, too) says that there exists $C_{0}>0$ such that

$$
\|x\| \leq C_{0}\|\delta x\|
$$


for all $\delta \in G(F)$ and $x \in \mathcal{S}_{0}^{\prime}$. Consequently, for those $x$,

$$
\left|B_{P, t}^{T}(x)\right| \leq\left(C_{0} C\right)^{t / c}\|x\|^{-t / c} \sum_{\delta \in P(F) \backslash G(F)} \hat{\tau}_{P}\left(H_{P}(\delta x)-T_{P}\right) .
$$

The sum occurring here is bounded by $C_{1}\|x\|^{c_{1}}$ for suitable $C_{1}, c_{1}$ by Lemma 5.1 of $[1]$.

If $t_{0} \leq t<0$, we start with a different estimate of the exponent. Namely, there exist $a_{0}>0$ and $b_{0}$ such that

$$
|H| \leq a_{0} \rho_{P}(H)+b_{0}
$$

for $H \in{ }^{+} \mathfrak{a}_{P}+T_{P}$. Assuming that $t_{0}<-2$, this allows us to estimate $B_{P, t_{0}}^{T}$ (and hence $B_{P, t}^{T}$ ) by an Eisenstein series, which is bounded by $C_{2}\|x\|^{c_{2}}$ for suitable $C_{2}$ and $c_{2}>0$.

Property (vi) of the height function (see [19]) allows us to reformulate our estimates in terms of the majorant figuring in the statement of the lemma, where we may use the Siegel domain $\mathcal{S}_{0}^{\prime}$ as well, since both sides are $P_{0}(F)$-invariant and $P_{0}(F) \mathcal{S}_{0}^{\prime}=P_{0}(F) \mathcal{S}_{0}$ for suitable $\omega_{0}$ and $T_{0}$. Both cases for the sign of $t$ can be combined in one inequality by choosing a suitable affine function of $t$ in the exponent.

\section{References}

[1] J. Arthur, A trace fomula for reductive groups I: Terms associated to classes in $G(\mathbf{Q})$, Duke Math. J. 45 (1978), 911-952

[2] J. Arthur, A trace fomula for reductive groups II: Applications of a truncation operator, Comp. Math. 40 (1980), 87-121

[3] J. Arthur, The trace formula in invariant form, Ann. of Math. 114 (1981), $1-74$

[4] J. Arthur, A measure on the unipotent variety, Canad. J. Math. 37 (1985), $1237-1274$

[5] A. Borel, T. A. Springer, Rationality properties of linear algebraic groups II, Tohoku Math. J. (2) 20 (1968), 443-487

[6] A. Deitmar, W. Hoffmann, Spectral estimates for towers of noncompact quotients, Canad. J. Math. 51 (1999), no. 2, 266-293

[7] L. D. Faddeev, The eigenfunction expansion of Laplace's operator on the fundamental domain of a discrete group on the Lobačevskii plane. Transactions of the Moscow Mathematical Society for the year 1967 (Volume 17). Amer. Math. Soc., Providence, R.I. 1969. 
[8] I. M. Gel'fand, I. I. Pyatetskiǔ-Šapiro, Avtomorfnye funktsii i teoriya predstavleniǔ, (Russian) Trudy Moskovskogo Mat. Obščestva 12 (1963), 389-412

[9] I. M. Gel'fand, M. I. Graev, I. I. Pyatetskii-Shapiro, Representation Theory and Automorphic Functions, B. W. Saunders Co., Philadelphia, 1969

[10] R. Godement, Introduction à la théorie de Langlands, Séminaire Bourbaki $321(1967 / 68)$

[11] Harish-Chandra, Discrete series for semisimple Lie groups II, Acta Math. 116 (1966), 1-111

[12] Harish-Chandra, Automorphic forms on semisimple Lie groups. Lecture Notes in Math., vol. 62, Springer, New York, 1968

[13] Harish-Chandra, Harmonic analysis on reductive p-adic groups, in: Harmonic Analysis on Homogeneous spaces, Proc. Symp. Pure Math., vol. 26, 1973, pp. 167-192

[14] B. Kostant, On convexity, the Weyl group and the Iwasawa decomposition, Ann. Sci. École Norm. Sup. 6 (1973), 413-455

[15] S. Lang, $S L(2, \mathbb{R})$. Addison-Wesley, Reading, 1975

[16] R. P. Langlands, Eisenstein series, in: Proc. Symp. Pure Math, vol. 9 (1966), Amer. Math. Soc., Providence, RI, p. 235-252

[17] R. P. Langlands, On the Functional Equations Satisfied by Eisenstein Series, Lecture Notes in Math., vol. 544, Springer, New York, 1976

[18] J. Levy, A truncated Poisson formula for groups of rank at most two, Amer. J. Math. 117 (1995), 1371-1408

[19] C. Moeglin, J.-L. Waldspurger, Spectral decomposition and Eisenstein series, Cambridge University Press, 1995

[20] W. Müller, On the spectral side of the Arthur trace formula, Geom. Funct. Anal. 12 (2002), 669-722

[21] M. S. Osborne, G. Warner, The Selberg trace formula I: $\Gamma$-rank one lattices, J. reine angew. Math. 3324 (1981), 1-113

[22] T. A. Springer, Reductive groups, in: Automorphic forms, representations an L-functions, Proc. Symp. Pure Math., vol. 33, part 1, Amer. Math. Soc., Providence, 1979

[23] A. Silberger, Introduction to harmonic analysis on reductive $p$-adic groups, Princeton Univ. Press, 1979 
[24] A. Silberger, Convexity for a simply connected $p$-adic group. Bull. Amer. Math. Soc. 81 (1975), 910-912.

[25] G. Warner, Harmonic analysis on semi-simple Lie groups, vol. II, Springer, New York 1972 